\title{
Characterisation of tau in the human and rodent enteric nervous system under physiological conditions and in tauopathy
}

Arthur Lionnet ${ }^{1,2,3}$, Matthew A. Wade ${ }^{4}$, Anne-Gaëlle Corbillé ${ }^{1,2,3}$, Alice Prigent ${ }^{1,3}$, Sébastien Paillusson ${ }^{4}$, Maddalena Tasselli ${ }^{4}$, Jacques Gonzales ${ }^{1,3}$, Emilie Durieu ${ }^{1}$, Malvyne Rolli-Derkinderen ${ }^{1,3}$, Emmanuel Coron ${ }^{1,3}$, Emilie Duchalais ${ }^{1,3}$, Michel Neunlist $^{1,3}$, Michael S. Perkinton ${ }^{5}$, Diane P. Hanger ${ }^{4}$, Wendy Noble ${ }^{4^{*}}$ (D) and Pascal Derkinderen ${ }^{1,2,3^{*}}$

\begin{abstract}
Tau is normally a highly soluble phosphoprotein found predominantly in neurons. Six different isoforms of tau are expressed in the adult human CNS. Under pathological conditions, phosphorylated tau aggregates are a defining feature of neurodegenerative disorders called tauopathies. Recent findings have suggested a potential role of the gut-brain axis in CNS homeostasis, and therefore we set out to examine the isoform profile and phosphorylation state of tau in the enteric nervous system (ENS) under physiological conditions and in tauopathies. Surgical specimens of human colon from controls, Parkinson's disease (PD) and progressive supranuclear palsy (PSP) patients were analyzed by Western Blot and immunohistochemistry using a panel of anti-tau antibodies. We found that adult human ENS primarily expresses two tau isoforms, localized in the cell bodies and neuronal processes. We did not observe any difference in the enteric tau isoform profile and phosphorylation state between PSP, PD and control subjects. The htau mouse model of tauopathy also expressed two main isoforms of human tau in the ENS, and there were no apparent differences in ENS tau localization or phosphorylation between wild-type and htau mice. Tau in both human and mouse ENS was found to be phosphorylated but poorly susceptible to dephosphorylation with lambda phosphatase. To investigate ENS tau phosphorylation further, primary cultures from rat enteric neurons, which express four isoforms of tau, were pharmacologically manipulated to show that ENS tau phosphorylation state can be regulated, at least in vitro. Our study is the first to characterize tau in the rodent and human ENS. As a whole, our findings provide a basis to unravel the functions of tau in the ENS and to further investigate the possibility of pathological changes in enteric neuropathies and tauopathies.
\end{abstract}

Keywords: Tau, Tau isoform, Tau phosphorylation, Enteric nervous system, Progressive supranuclear palsy, Parkinson's disease, Gut, Biopsy, Htau mouse

\footnotetext{
* Correspondence: Wendy.Noble@kcl.ac.uk; pascal.derkinderen@chu-nantes.fr; derkinderenp@yahoo.fr

Arthur Lionnet, Matthew A. Wade, Anne-Gaëlle Corbillé and Alice Prigent contributed equally to this work.

Wendy Noble and Pascal Derkinderen Co-senior authorship

${ }^{4}$ King's College London, Institute of Psychiatry, Psychology and Neuroscience,

Department of Basic and Clinical Neuroscience, Maurice Wohl Clinical

Neuroscience Institute, Rm 1.23, 5 Cutcombe Road, Camberwell, London SE5

$9 \mathrm{RX}, \mathrm{UK}$

${ }^{1}$ Inserm, U1235, 1 rue Gaston Veil, F-44035 Nantes, France

Full list of author information is available at the end of the article
}

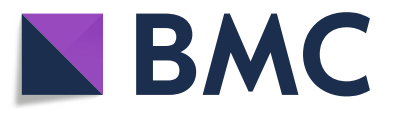

(c) The Author(s). 2018 Open Access This article is distributed under the terms of the Creative Commons Attribution 4.0 International License (http://creativecommons.org/licenses/by/4.0/), which permits unrestricted use, distribution, and reproduction in any medium, provided you give appropriate credit to the original author(s) and the source, provide a link to the Creative Commons license, and indicate if changes were made. The Creative Commons Public Domain Dedication waiver (http://creativecommons.org/publicdomain/zero/1.0/) applies to the data made available in this article, unless otherwise stated. 


\section{Introduction}

The microtubule-associated protein tau is found predominantly in neurons, where it exists as a highly soluble protein that interacts with the cytoskeleton [25, 28]. Six different isoforms of tau are expressed in the adult human CNS via alternative splicing of the $M A P T$ gene, which comprises 16 exons. Regulated inclusion of exons 2 and 3 yields tau isoforms with 0,1 , or $2 \mathrm{~N}$-terminal inserts $(0 \mathrm{~N}$, $1 \mathrm{~N}, 2 \mathrm{~N}$, respectively), whereas exclusion or inclusion of exon 10 leads to expression of tau isoforms with three (3R) or four (4R) microtubule-binding repeats [28]. The various splice combinations of tau are thus abbreviated 0N3R, 0N4R, 1N3R, 1N4R, 2N3R, 2N4R, encoding six protein isoforms ranging from 352 to 441 amino acids in length [25]. The function of tau is strongly affected by its phosphorylation status, which influences its ability to interact with microtubules and various signaling proteins [20,57], as well as its localization and association with membranes $[56,63]$. Under pathological conditions, aberrant assembly of highly phosphorylated tau into insoluble aggregates is observed in a range of neurodegenerative disorders, collectively referred to as tauopathies. Tauopathies encompass more than 20 clinicopathological entities, including Alzheimer's disease (AD), progressive supranuclear palsy (PSP), Pick's disease, all of which can be biochemically subclassified according to the predominance of tau isoforms found in the intracellular aggregates [43]. Tau aggregates found in tauopathies generally contain tau in an elevated state of phosphorylation [7, 29, 34] that is often aberrantly cleaved [31, 51]. Highly phosphorylated forms of tau are also found in other neurodegenerative diseases, including Parkinson's disease (PD), where it often colocalises with abnormal alpha-synuclein $[39,66]$.

The enteric nervous system (ENS) is an integrated neuronal network distributed from the lower esophagus to the rectum. Compared to other components of the peripheral nervous system, the ENS shows some unique features that closely resemble the CNS and is sometimes referred to as 'the brain-in-the-gut' or the 'second brain'. This close homology between the CNS and ENS suggests that a disease process affecting the CNS could also involve its enteric counterpart, as has already been described in variant Creutzfeldt-Jakob disease [33, 41] and PD [6, 21, 65]. Whether such a scenario can be extended to other neurodegenerative disorders such as tauopathies remains to be demonstrated, and this was one focus of the current study.

A few studies have shown that tau is expressed in rodent [30] and human $[8,17,61]$ gastrointestinal (GI) tract, but no data are available about the distribution and phosphorylation pattern of tau isoforms in the ENS. Here, we examined the expression levels of tau isoforms, their phosphorylation profile and truncation in sigmoid colon biopsy specimens from PSP patients and compared them to samples from PD patients and controls. We examined the same tau characteristics in a mouse model of tauopathy in comparison to wild-type mice. Our results show the expression of two main human tau isoforms in the ENS. ENS tau is phosphorylated but is remarkably resistant to dephosphorylation with lambda phosphatase. We then examined the isoform profile and phosphorylation state of tau under physiological conditions in rat primary enteric neuron cultures, which showed that ENS tau phosphorylation can be modified, at least in vitro. These data provide the first detailed characterization of ENS tau in humans and rodents in health and tauopathies. Further investigation of tau modifications in the ENS in disease may provide valuable information about tau modifications that promote or prevent tau abnormalities spreading between the gut and brain in neurodegenerative diseases.

\section{Material and methods}

\section{Human tissues}

Samples of frozen temporal cortex from one post-mortem human brain devoid of neurodegeneration were obtained from the Neuropathology Department of Angers (Dr Franck Letournel) to serve as a control for the following experiments. Specimens of human colon were obtained from three neurologically unimpaired subjects who underwent colon resection for colorectal cancer. For all three tissues specimens, sampling was performed in macroscopically normal segments of uninvolved resection margins. Colonic sections were separated into muscle and submucosal/mucosal layer [36], which contain the myenteric and submucosal plexus respectively. Two out of three samples were frozen and kept at $-80{ }^{\circ} \mathrm{C}$ until further analysis by Western blot. The remaining sample was analyzed by immunohistochemistry.

Routine sigmoid colon biopsies were obtained during sigmoidoscopy/colonoscopy from 24 subjects, 10 with PD, 5 with PSP and 9 controls. All patients were recruited from the movement disorder clinic at Nantes University Hospital, France. Diagnosis of PD was made according to criteria provided by the United Kingdom Parkinson's Disease Survey Brain Bank. PSP patients fulfilled the diagnostic criteria for possible or probable PSP. Control subjects were healthy subjects who had a routine colonoscopy performed for colorectal cancer screening. All controls subjects underwent a detailed neurological examination to rule out PD symptoms and cognitive deficiency. Except for control subjects 183 and 208 (Table 1) who had 6 biopsies, 4 biopsies per patient were taken during the endoscopic procedure. Biopsies were stored at $-80{ }^{\circ} \mathrm{C}$ until required.

The sampling of human brain and colon was approved by the Fédération des biothèques of the University Hospital of Nantes, according to the guidelines of the French Ethics Committee for Research on Humans and registered under the no. DC-2008-402. Regarding sigmoid biopsies sampling, 
Table 1 Demographics and characteristics of controls subjects and patients

\begin{tabular}{|c|c|c|c|}
\hline Patient \# & Age/sex & Diagnosis & $\mathrm{DD}$ \\
\hline 183 & $49 / F$ & Control & - \\
\hline 188 & $67 / F$ & Control & - \\
\hline 189 & $63 / F$ & Control & - \\
\hline 190 & $45 / M$ & Control & - \\
\hline 191 & $19 / F$ & Control & - \\
\hline 208 & $76 / \mathrm{M}$ & Control & - \\
\hline 210 & $63 / F$ & Control & - \\
\hline 214 & $69 / F$ & Control & - \\
\hline 227 & $56 / F$ & Control & - \\
\hline 162 & $56 / F$ & PD & 12 \\
\hline 166 & $64 / F$ & PD & 11 \\
\hline 167 & $67 / M$ & PD & 10 \\
\hline 168 & $55 / F$ & PD & 4 \\
\hline 171 & $71 / M$ & PD & 3 \\
\hline 173 & $67 / M$ & PD & 11 \\
\hline 175 & $70 / \mathrm{M}$ & PD & 12 \\
\hline 177 & $70 / F$ & PD & 8 \\
\hline 178 & $53 / F$ & PD & 1 \\
\hline 179 & $52 / F$ & PD & 4 \\
\hline 170 & $63 / F$ & PSP & 4 \\
\hline 176 & $72 / \mathrm{M}$ & PSP & 4 \\
\hline 185 & $72 / F$ & PSP & 11 \\
\hline 187 & $75 / \mathrm{M}$ & PSP & 5 \\
\hline 228 & $76 / F$ & PSP & 1 \\
\hline
\end{tabular}

Patient ID, age, sex, diagnosis of PD or PSP (including probable PSP) are shown in addition to disease duration (DD) in years

the study protocol was approved by the local Committee on Ethics and Human Research (Comité de Protection des Personnes Ouest VI), and registered on ClinicalTrials.gov (EnteroLark and ColoBioParker, identifier NCT01618383 and NCT01353183, respectively). Written informed consent was obtained from each patient and from each normal volunteer.

\section{Mouse tissues}

Htau mice (B6.Cg-Mapt ${ }^{\text {tm1(EGFP)Klt }} \mathrm{Tg}$ (MAPT)8cPdav/J) were originally purchased from the Jackson laboratory (Bar Harbor, ME, USA) and maintained at King's College London. Wild-type and tau knockout offspring of an identical background strain $(\mathrm{C} 57 \mathrm{Bl} / 6 \mathrm{~J})$ were obtained via breeding. All housing and experimental procedures were carried out in compliance with the local ethical review panel of King's College London under a UK Home Office project license held in accordance with the Animals (Scientific Procedures) Act 1986 and the European Directive 2010/63/EU. Two-month old male and female mice were used in this study. Animals were housed at $19-22{ }^{\circ} \mathrm{C}$, humidity 55\%, $12 \mathrm{~h}: 12 \mathrm{~h}$ light: dark cycle with lights on at 07:30. Animals were culled using Schedule 1 methods, brains removed and snap-frozen on dry-ice. Sections of colon tissue were removed, with tissue from the distal portion of each part being cleaned and snap-frozen on dry-ice, prior to storage at $-80{ }^{\circ} \mathrm{C}$ for RNA extraction or biochemical analysis. The proximal portion from each part of colon along with the duodenum, jejunum and ileum were dissected with fine forceps to reveal the myenteric plexus as described previously [62].

\section{Rat tissues}

Sciatic nerve sections were taken from two pregnant Sprague-Dawley rats (used for the generation of primary culture of rat ENS, see below) to serve as a positive control for big tau experiments [60].

\section{Primary cultures of rat ENS}

Primary culture of rat ENS were generated using pregnant Sprague-Dawley rats (Janvier Laboratories SA, Le Genest-St-Isle, France) as previously described [11]. All housing and experimental procedures were carried out in compliance with the local ethical review panel of INSERM (agreement E. 44,011; INSERM, Nantes, France). Pregnant rats were killed by an overdose of $\mathrm{CO}_{2}$ followed by severing the carotid arteries. The small intestines of rat embryos were removed, diced in Hank's Buffered Salt Solution (Sigma, Saint-Quentin Fallavier, France) and collected in $5 \mathrm{~mL}$ of Dulbecco's modified Eagle's medium (DMEM)-F12 (Gibco ${ }^{\circ}$, Life Technologies, Villebon sur Yvette, France) (1:1) for digestion at $37^{\circ} \mathrm{C}$ for $15 \mathrm{~min}$ in $0.1 \%(v / v)$ trypsin (Sigma). The trypsin reaction was stopped by adding medium containing $10 \%$ fetal calf serum and then treatment with DNase I $0.01 \%(\mathrm{v} / \mathrm{v})$ (Sigma) for $10 \mathrm{~min}$ at $37^{\circ} \mathrm{C}$. After triturating with a $10 \mathrm{~mL}$ pipette, cells were centrifuged at $750 \mathrm{rpm}$ for $10 \mathrm{~min}$. Cells were counted and then seeded at a density of $2.4 \times 10^{5} \mathrm{cells} / \mathrm{cm}^{2}$ on 24-well plates previously coated with a solution of $0.5 \%$ $(\mathrm{v} / \mathrm{v})$ gelatin in sterile phosphate buffered saline. After $24 \mathrm{~h}$, the medium was replaced with a serum-free medium DMEM-F12 (1:1) containing 1\% (v/v) of N-2 supplement (Life Technologies). Cultures were maintained for 14 days.

\section{Treatment of rat ENS primary cultures with serine/ threonine phosphatases inhibitors}

After 14 days in vitro (DIV), cells were treated with a cocktail of three phosphatase inhibitors including $1 \mu \mathrm{M}$ okadaic acid, $1 \mu \mathrm{M}$ ciclosporine A and $6.75 \mu \mathrm{M}$ sanguinarine (Sigma) for broad-spectrum inhibition of serine/ threonine phosphatases, or with vehicle (DMSO, Sigma) for one hour. 


\section{Dephosphorylation of tissues and cell lysates}

For dephosphorylation experiments, cells or tissues were homogenised in a buffer containing $100 \mathrm{mM} \mathrm{NaCl}$ and $50 \mathrm{mM}$ Tris-Cl at $\mathrm{pH} 7.4$ with $1 \%$ (v/v) IGEPAL ${ }^{\circ} \mathrm{CA}-630$ and a protease inhibitors cocktail without EDTA (Roche, Neuilly sur Seine, France) using either a "Precellys 24" (Bertin technologies, St Quentin-en-Yvelines, France) or a Tissue Master 125 (Omni International, Kennesaw, GA, USA) tissue homogenizer and followed by sonication with "vibracell 75 186" device (Sonics, Newton CT, USA). Homogenates were centrifuged at $16,300 \mathrm{~g}$ for $20 \mathrm{~min}$ at $4{ }^{\circ} \mathrm{C}$ with an Eppendorf 5415R centrifuge (Eppendorf, Hamburg, Germany), sonicated for $10 \mathrm{~s}$ and protein amounts normalized following a BCA protein assay (ThermoFisher, Waltham, MA, USA). Samples were diluted to $1.0 \mathrm{mg} / \mathrm{mL}$ protein using homogenisation buffer and incubated with $20 \mathrm{U} / \mu \mathrm{L}$ lambda phosphatase in $\mathrm{MnCl}_{2}$ and enzyme buffer as supplied with the lambda protein phosphatase kit (New England Biolabs, Ipswich, MA, USA) for $3 \mathrm{~h}$ at $30{ }^{\circ} \mathrm{C}$. The reaction was stopped by the addition of sample buffer (National Diagnostics, Hull, UK or Life Technologies, Courtaboeuf, France) and heating to $95{ }^{\circ} \mathrm{C}$ for $5 \mathrm{~min}$. Control samples were treated identically without the addition of lambda phosphatase.

\section{SDS-PAGE and western blot}

For dephosphorylation experiments, cells or tissues were processed as described above. For experiments that did not require dephosphorylation, cells or tissues were lysed in RIPA lysis buffer (Merck Millipore, Fontenay sous Bois, France). Western blots were performed as we previously described [10] using NuPAGE ${ }^{\mathrm{max}} 10 \%$ Bis-Tris Protein Gels (Life Technologies, Courtaboeuf, France). The primary anti-tau antibodies used are listed in Table 2.
Phospho-ERK (Cell signaling, Ozyme, France 1:2000 dilution) and PGP 9.5 antibodies (Abcam, France, 1:1000 dilution) were used for the evaluation of phosphatase treatment and as loading control, respectively.

\section{Immunohistochemistry}

For mouse GI tract tissues, following the excision of myenteric plexus from mouse colon, tissue segments were incubated in combined blocking $(50 \mathrm{mM}$ tris-buffered saline [TBS] pH 7.4 containing $5 \%$ bovine serum albumen [BSA] and $0.05 \%$ tween-20) and permeabilisation $(50 \mathrm{mM}$ TBS pH 7.4, 0.1\% triton X-100) solutions overnight at $4{ }^{\circ} \mathrm{C}$. Primary antibodies (Table 2) in blocking solution were incubated with gut tissues overnight at $4{ }^{\circ} \mathrm{C}$. Following washing in $50 \mathrm{mM}$ TBS the appropriate fluorescently-tagged secondary antibody was added for $3 \mathrm{~h}$ at ambient temperature, the antibodies removed by washing and Hoechst 33258 added for $3 \mathrm{~min}$. Images were acquired using a CTR5000 digital camera (Leica Microsystems, Cambridge, UK) attached to a Leica DM5000B fluorescence microscope with Leica AIF lite software.

For human tissues, fixed human tissues were embedded in paraffin using an embedding station (LEICA EG1150C) and sections $(3 \mu \mathrm{m})$ were cut using a microtome (LEICA RM2255). The sections were deparaffinised by bathing twice in xylene (for 5 min each) and taken through graded concentrations of ethanol (100, $95,70,70 \%$, respectively for 3 min each). After a rinse in distilled water, slides were washed in PBS and antigen retrieval was performed using a sodium citrate solution (2.94 g Sodium Citrate Tribase; $1 \mathrm{~L}$ ultrapure water; $500 \mu \mathrm{L}$ Tween 20; pH 6) at $95{ }^{\circ} \mathrm{C}$ for $20 \mathrm{~min}$. Slides were incubated in $\mathrm{NH}_{4} \mathrm{Cl}(100 \mathrm{mM})$ for $15 \mathrm{~min}$ before incubation in PBS- $0.5 \%$ triton $\mathrm{X}-100$ for $1 \mathrm{~h}$ and blocking for

Table 2 Tau antibodies used in this study

\begin{tabular}{|c|c|c|c|}
\hline Name & Specificity & Epitope (a.a) & Source and dilution \\
\hline A0024 Tau & All tau isoforms & $243-441$ (2N4R) & Dako, rp (WB 1:1000; IHC 1:500) \\
\hline TAU-5 & All tau isoforms & $210-241$ (2N4R) & ThermoFisher, mm (WB 1:1000) \\
\hline Tau-1 & All tau isoforms & 189-207 (2N4R) & Merck, mm, clone PC1C6 (WB 1:2000) \\
\hline TP70 & All tau isoforms & $428-441$ (2N4R) & IOP, KCL, rp (WB 1:500) \\
\hline Anti tau RD3 & 3R tau Isoforms & $267-282(2 N 3 R)$ & Merck, mm, clone 8E6 (WB 1:1000; IHC 1:500) \\
\hline Anti tau RD4 & $4 \mathrm{R}$ tau isoforms & 275-291 (2N4R) & Merck, mm, clone 1E1/A6 (WB 1:1000) \\
\hline Anti 4R-tau & $4 \mathrm{R}$ tau isoforms & NS & Cosmo bio co., rp (WB 1:2000; IHC 1:1000) \\
\hline Anti $0 \mathrm{~N}$-tau & O N Tau isoforms & 39-50 (ON3R) & BioLegend, mm (WB 1:500) \\
\hline AT8 & Tau @ S202/T205 & Tau @ S202/T205 & Innogenetics, mm (WB 1:1000) \\
\hline PHF-1 & Tau @ S396/S404 & Tau $\odot$ S396/S404 & Gift from Peter Davies, mm (WB 1:500) \\
\hline PHF13 & Tau @ S396 & Tau @ S396 & Cell Signaling, mm (WB 1:1000) \\
\hline CP13 & Tau @ S202 & Tau @ S202 & Gift from Peter Davies, mm (IHC 1:200) \\
\hline
\end{tabular}

The name, specificity, epitope, source and dilution of the antibodies used in this study are shown.

Abbreviations: a.a. amino-acids, IHC immunohistochemistry, IOP, $K C L$ Institute of Psychiatry, King's college London, mm mouse monoclonal, $N S$ not specified, $r p$ rabbit polyclonal, WB western blot 
$2 \mathrm{~h}$ in $10 \%$ horse serum in PBS- $0.5 \%$ triton X-100. Primary antibodies (Table 2) were incubated overnight at $4{ }^{\circ} \mathrm{C}$, and following washing, secondary antibodies were added for $2 \mathrm{~h}$ at room temperature. Images were acquired with an Olympus IX 50 fluorescence microscope coupled to a digital camera (model DP71, Olympus).

\section{RNA extraction and RT-PCR}

Frozen proximal colon and cortex from htau, wild-type and tau knockout mice was homogenised in approximately $100 \mathrm{mg} / \mathrm{mL}$ Quiazol $^{\circ}$ supplied with the Quiagen RNA LipidEasy kit (Qiagen, Hilden, Germany), and RNA was extracted following the manufacturer's protocol. The RNA obtained was eluted in ultrapure $\mathrm{H}_{2} \mathrm{O}$ and its concentration and purity determined using a NanoDrop spectrophotometer (Thermo Scientific, Waltham, MA, USA). Samples were diluted to $1 \mu \mathrm{g}$ RNA/15 $\mu \mathrm{L}$ RNAse-free $\mathrm{H}_{2} \mathrm{O}$, heat-shocked for $3 \mathrm{~min}$ at $72{ }^{\circ} \mathrm{C}$ to break down double-stranded structures and returned immediately to ice. One $\mu$ g RNA per sample was reverse transcribed using a Superscript III reverse transcriptase assay kit (Life Technologies, Paisley, UK) according to the manufacturer's instructions. The resulting cDNA was stored at $-20{ }^{\circ} \mathrm{C}$ until use. To examine the alternate splicing of the microtubule binding domain repeat region encoded by exon 10, primers were used that specifically recognize mouse or human exons 9 and 11 as described by Duff et al. [16]. Primer sequences were: mouse exon 9F 5'-CCCCCTAAGTCACCATCAGCTAGT, mouse exon 11R 5'-CACTTTGCTCAGGTCCACCGGC, human exon 9F 5'-CTCCAAAATCAGGGGATCGC, human exon 11R 5'-CCTTGCTCAGGTCAACTGGT. Splicing around the $\mathrm{N}$ terminal insert domain encoded by exons 2 and 3 was detected using primers that recognize exons 1 and 5 . Primer sequences used were: mouse exon 1F 5'-TCCG CTGTCCTCTTCTGTC, mouse exon 5R 5' - TTCTCG TCATTTCCTGTCC, human exon 1F 5' - TGAACCA GGATGGCTGAGC, human exon 5R 5'-TTGTCATCG CTTCCAGTCC. Annealing temperatures were $64{ }^{\circ} \mathrm{C}$ (all $M A P T$ primers), $62{ }^{\circ} \mathrm{C}$ (M1F/M5R) and $68{ }^{\circ} \mathrm{C}$ (M9F/ M11R). 35 reaction cycles were used for all. Mouse and human-specific RT-PCR products were analysed by agarose gel electrophoresis. Products corresponding to exon 10+ tau mRNA (4R) are 390 base pairs (bp), while products corresponding to exon 10- mRNA (3R) are $297 \mathrm{bp}$. RT-PCR products containing tau mRNA with exons 2 and $3(2 \mathrm{~N})$ are $428 \mathrm{bp}, 2+3-$ mRNA products $(1 \mathrm{~N})$ are $341 \mathrm{bp}$, and 2-3- mRNA products $(0 \mathrm{~N})$ are $253 \mathrm{bp}$.

\section{Statistics}

All data shown are mean \pm SEM. Statistical analyses was conducted using GraphPad software version 5.00 (San Diego California, USA). For comparisons of means between groups, Kruskal-Wallis tests were performed. Differences were deemed statistically significant when $p<0.05$.

\section{Results \\ The expression pattern of tau isoforms is different in adult human brain and gut}

In adult human brain, the six tau isoforms are phosphorylated resulting in reduced electrophoretic mobility on SDS-PAGE compared to recombinant tau [25]. In order to identify the tau isoforms expressed in the human ENS, colonic samples from healthy subjects treated or not with lambda phosphatase [35] were analyzed by western blot using the A0024 tau antibody that recognizes all six tau isoforms. ENS samples were compared to dephosphorylated and non-dephosphorylated brain samples as well as to a recombinant tau ladder. The banding pattern was markedly different between brain and colonic samples (Fig. 1a). The A0024 Tau antibody detected one major band migrating at $53-54 \mathrm{kDa}$ in both the submucosal and muscle layers (which contain the submucosal and myenteric plexus, respectively and therefore are referred to as SMP and MP) (Fig. 1a). This band migrated only slightly faster after dephosphorylation of SMP and MP samples despite the efficiency of the dephosphorylation treatment being validated by phospho-ERK immunoblot, Fig. 1a). The major band detected in ENS samples comigrated with 0N4R-1N3R detected in human brain samples and the recombinant tau ladder (red line in Fig. 1a) and was also observed when a pan-tau (TAU-5) antibody was used (Fig. 1b). In addition, a fainter band around $57-58 \mathrm{kDa}$ in SMP and a strong immunoreactive band at $62 \mathrm{kDa}$ (white arrow) in both SMP and MP were also observed when the A0024 tau antibody was used (Fig. 1a). These two bands are most likely non-specific as they were not observed with TAU-5 (Fig. 1b) or with other specific antibodies subsequently used in this study (Figs. 1b, c and 2).

To further refine this analysis, we used 3 commercially available isoform-specific tau antibodies. Two of these antibodies directed against $3 \mathrm{R}$ and $0 \mathrm{~N}$-tau have been shown to be highly specific in a recent comprehensive study that tested the specificity of tau antibodies using immunoblotting [19]. In addition, we used a 4R-tau antibody that only detects $4 \mathrm{R}$ tau isoforms in human brain lysates and in tau ladder (Additional file 1: Figure S1). All of these antibodies detected a single 53-54 kDa-band that comigrates with the major band detected by TAU- 5 and with 0N4R-1N3R in the recombinant tau ladder (Fig. 1b).

Until recently, analysis of the ENS in humans was mainly performed using full thickness specimens of the gut obtained during surgery or autopsy. However, several recent studies have shown that the ENS is accessible and analyzable through routine GI biopsies, which can be processed to measure quantitative differences in neuronal 


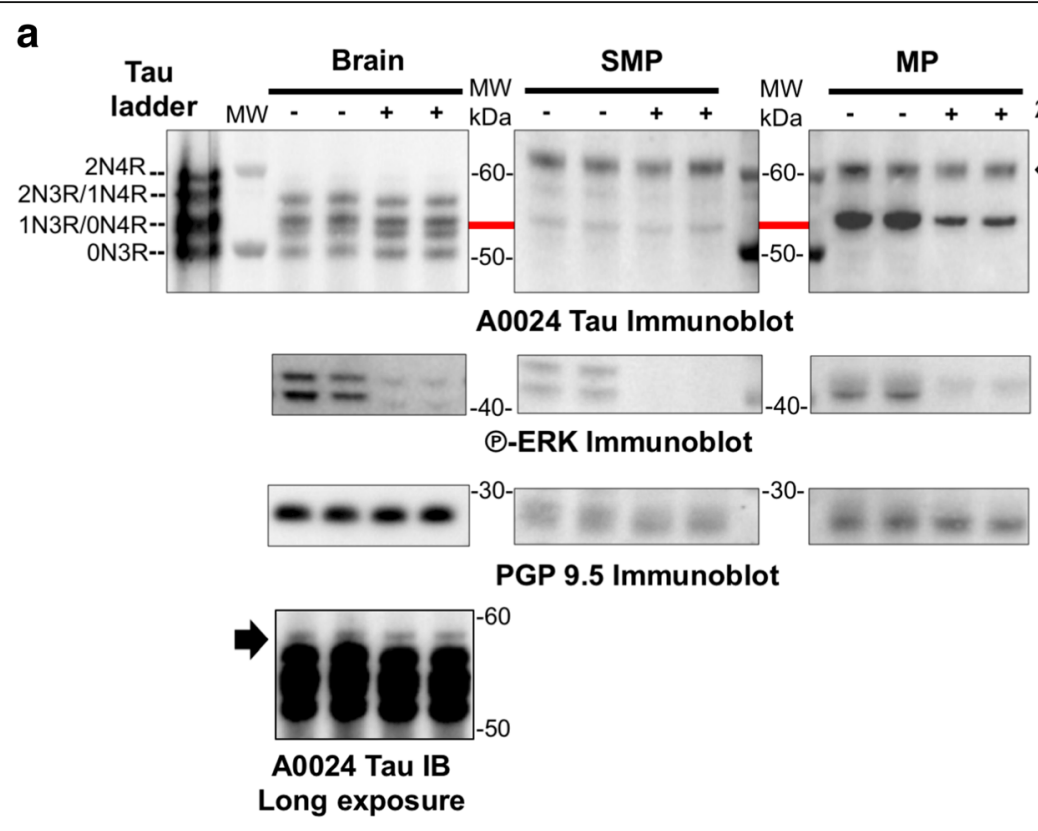

b

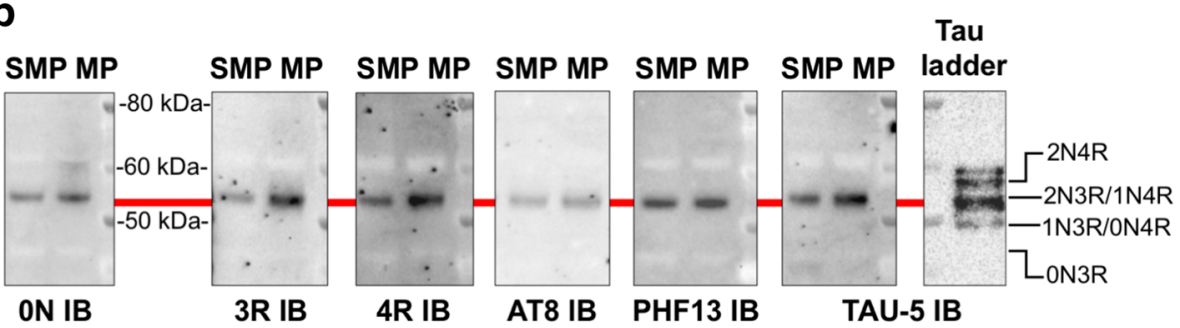

C

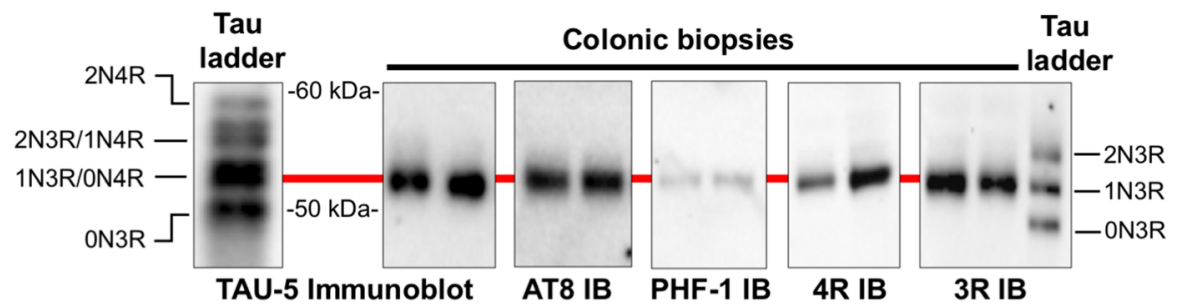

Fig. 1 Tau isoforms and phosphorylation in adult human ENS. a Human brain and colon tissue lysates (submucosal and muscle layers, which contains the submucosal (SMP) and myenteric plexus (MP), respectively) were subjected to immunoblot analysis using the pan-Tau antibody A0024. Lysates were treated (+) or not (-) with lambda phosphatase before immunoblotting. The effectiveness of dephosphorylation was confirmed by phospho-ERK immunoblot (P-ERK immunoblot). Tau antibody A0024 detected all six tau isoforms in the recombinant human tau ladder and brain samples (the 2N4R was only visible on long exposure immunoblots, black arrow). The non-specific band detected by Tau antibody A0024 in the ENS is marked by a white arrow. An antibody against protein gene product (PGP) 9.5 was used as a loading control. b Colon tissue lysates (SMP and MP) were subjected to immunoblot analysis using antibodies specific to 0 N, 3R, 4R tau, the pan-tau TAU-5 antibody, and the phospho-specific tau antibodies AT8 (phos-Ser202/Thr205) and PHF13 (phos-Ser396). c Sigmoid colon biopsies lysates from 2 control subjects (\#183 and 208, Table 2) were subjected to immunoblot analysis using the TAU-5 antibody, antibodies specific to the 3R and 4R tau isoforms and the phospho-specific tau antibodies AT8 and PHF-1. In all experiments, the banding pattern was compared to that of tau ladder which contains all six recombinant tau isoforms. The red line shows the comigration of the observed bands with 1 N3/0N4R. The results shown in (a), (b) and (c) are representative of 3, 2 and 5 independent experiments, respectively

and/or glial markers $[5,23,44]$. We therefore analyzed the expression levels of tau in routine sigmoid biopsies from 2 control subjects (\#183 and 208, Table 1) with the pan-tau antibody TAU-5 and with the $3 \mathrm{R}$ and $4 \mathrm{R}$ isoform-specific antibodies. The immunoblotting pattern observed with these 3 antibodies in biopsies was similar to those observed in colonic SMP and MP samples (Fig. 1c).

"Big" or peripheral tau is a tau isoform specifically expressed in the peripheral nervous system, including trigeminal, dorsal root and sympathetic ganglia as well as 


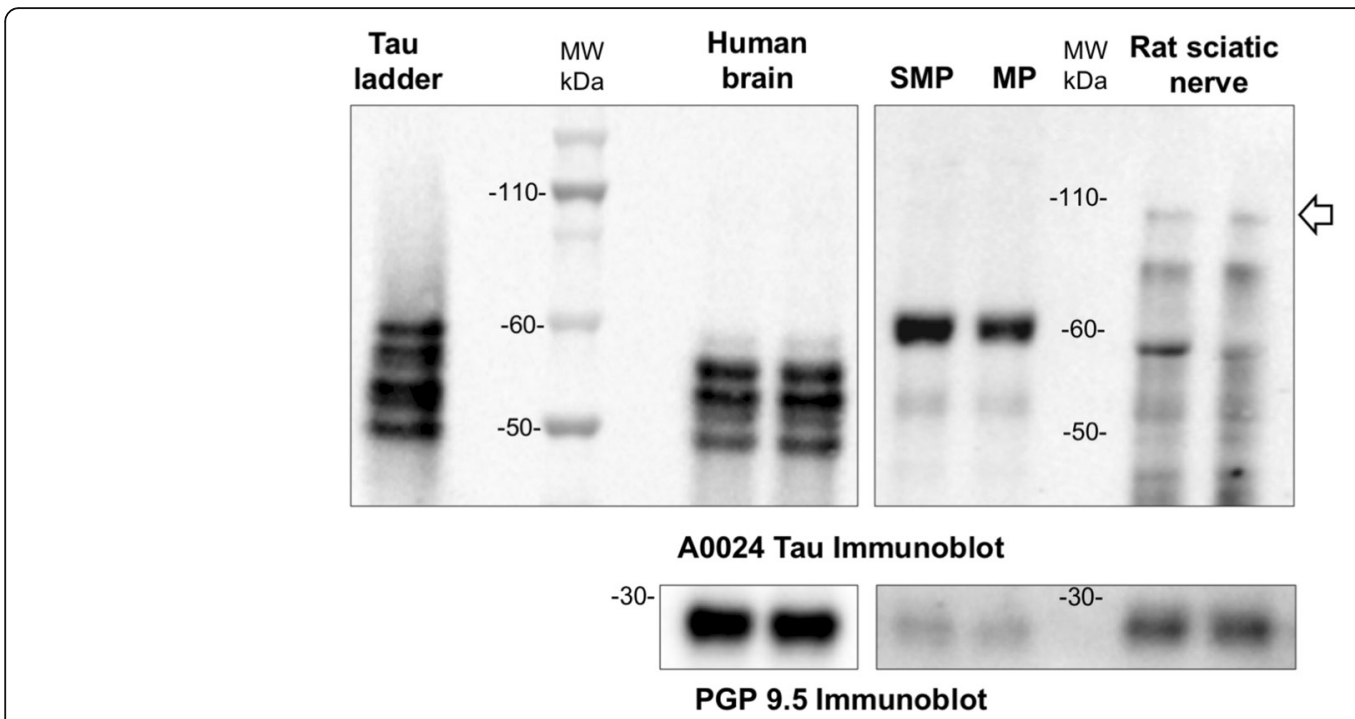

Fig. 2 Big tau is not detected in adult human ENS. Human brain and colon tissue lysates (SMP and MP) were subjected to immunoblot analysis using the pan-Tau antibody A0024. Rat sciatic nerve lysates were used as positive control to detect big tau (white arrow). PGP 9.5 was used as a loading control. Images are representative of five independent experiments

sciatic nerve. It differs from the $2 \mathrm{~N} 4 \mathrm{R}$ tau isoform by a 254 amino-acid insert located in the amino-terminal half and migrates at $110 \mathrm{kDa}$ on SDS/PAGE [27]. To determine whether big tau is expressed in the ENS, human colon tissue lysates were analyzed by Western blot using Tau A0024 antibody. Rat sciatic nerve lysates were used as positive controls [60]. Tau A0024 detected the expected low molecular weight tau isoforms between 45 and $60 \mathrm{kDa}$ in human colon and rat sciatic nerve, however a $110 \mathrm{kDa}$ migrating band was only observed with rat sciatic nerve lysates (Fig. 2).

When taken together, these results show that $1 \mathrm{~N} 3 \mathrm{R}$ and $0 \mathrm{~N} 4 \mathrm{R}$ are the two main tau isoforms that are expressed in human adult colon and these two isoforms can be detected in routine GI biopsies. In addition, our work indicates that big tau is not expressed in the adult human ENS.

\section{Tau isoforms are differentially expressed in the gut and brain of tauopathy mice}

To determine if tau is also differentially expressed in the ENS and CNS of mice, we used the transgenic htau mouse model which expresses exclusively the six wild-type human isoforms of tau under the control of the MAPT promoter [3]. The enteric expression profile of tau isoforms in these transgenic mice was compared to that observed in wild-type mice. Tau knockout mice were examined as an additional control. To this end, RNA from 2-month-old, wild-type, tau knockout and htau mouse proximal colon was reverse transcribed to cDNA and amplified with PCR. Brain tissue from the same mice was used for comparison. Primers were designed, based on those previously described by Duff et al. [16], to detect splicing of human tau exons 2 and 3. This allowed amplification of products corresponding to $0 \mathrm{~N}, 1 \mathrm{~N}$ and $2 \mathrm{~N}$ human tau that were detected in htau, but not wild-type or tau knockout, brain and proximal colon (Fig. 3). Transcripts of $3 \mathrm{R}$ and $4 \mathrm{R} M A P T$ were also observed in htau brain and proximal colon when inclusion of exon 10 was assessed using primers specific to human tau exons 9 and 11 (Fig. 3). Thus, $0 \mathrm{~N}, 1 \mathrm{~N}, 2 \mathrm{~N}, 3 \mathrm{R}$ and $4 \mathrm{R}$ human tau transcripts are expressed in htau proximal colon.

Primers against mouse tau were also used to allow detection of $0 \mathrm{~N}, 1 \mathrm{~N}$ and $2 \mathrm{~N}$ transcripts in wild-type mouse brain and proximal colon (Fig. 3a). A weak non-specific PCR product corresponding to the predicted size of $0 \mathrm{~N}$ tau was also amplified in htau and tau knockout samples with these primers. In addition, $4 \mathrm{R}$, but not $3 \mathrm{R}$ Mapt was detected in WT mouse brain, and a weak signal was also apparent in proximal colon (Fig. 3a). These transcripts were not amplified in htau or tau knockout tissues. Thus, wild-type mice express $0 \mathrm{~N}, 1 \mathrm{~N}, 2 \mathrm{~N}$ and mainly $4 \mathrm{R}$ tau in gut and brain.

The detection of multiple products in a single lane, each corresponding to a different tau isoform, allows each product to act as an internal control for the other transcripts. This allowed us to make comparisons between the relative abundance of tau isoforms in different tissues. Htau brain showed $0 N>1 N>2 \mathrm{~N}$ relative abundance of tau isoforms, in keeping with previous observations in adult mice [59]. Htau mouse brain also showed greater exclusion of tau exon $10(3 R>4 R)$, as previously reported [3] and in contrast to adult wild-type mice where mainly 4R tau is expressed (Fig. 3; [59]). Thus, htau brain mirrors human brain in that both $3 \mathrm{R}$ and $4 \mathrm{R}$ tau are expressed, 

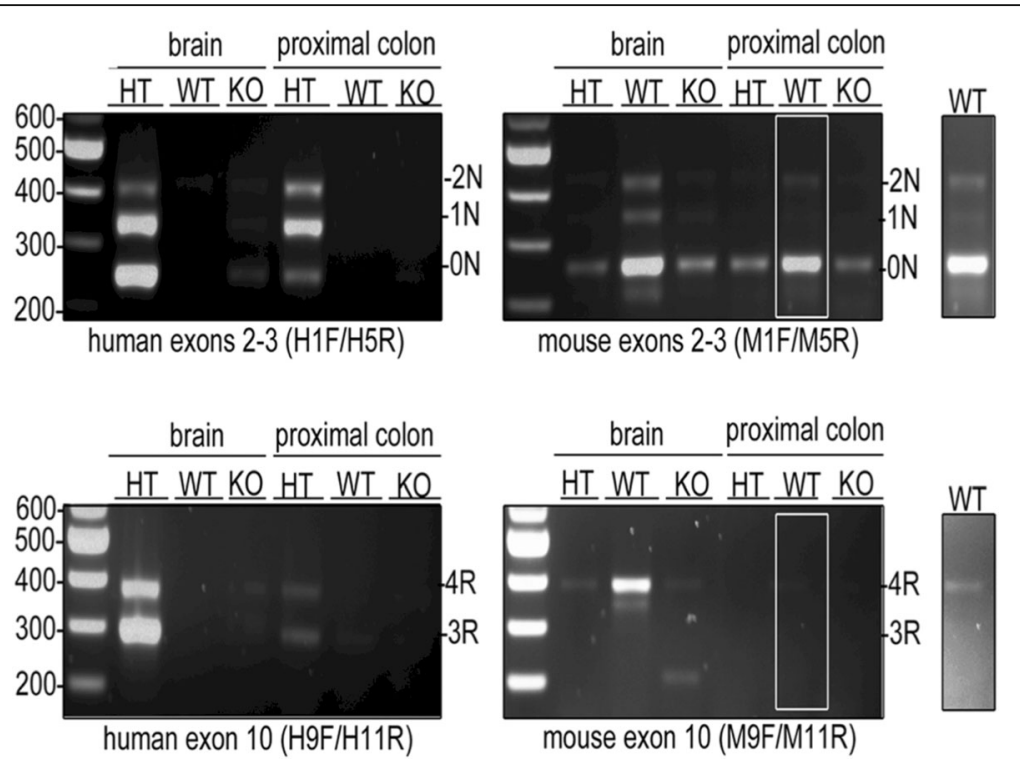

Fig. 3 Detection of tau in htau and wild-type mouse brain and proximal colon. Htau (HT), wild-type (WT) and tau knock-out (KO) brain and proximal colon CDNA was amplified using PCR with human- and mouse-tau specific primers to detect the expression of exon 2-3- (0 N), $2+3-(1 \mathrm{~N}), 2+3+(2 \mathrm{~N}), 10-(3 R)$ and 10+ (4R) tau isoforms. Gel images show detection of human and mouse $0 \mathrm{~N}, 1 \mathrm{~N}$ and $2 \mathrm{~N}$ tau. An insert shows a higher intensity portion of image to illustrate tau products in proximal colon. Numbers correspond to base pairs of a DNA ladder. The expected position of PCR product is indicated to the right of each panel. $N=3$

albeit that under physiological conditions these isoforms are expressed in approximately equal proportions in the human CNS [4]. In contrast, in htau proximal colon, PCR transcripts showed an altered relative abundance of $1 \mathrm{~N}>$ $2 N>0 \mathrm{~N}$ (Fig. 3), and there appeared to be approximately equal inclusion and exclusion of exon $10(3 R \approx 4 R)$. These data suggest differential expression of tau isoforms in the ENS of htau mice in comparison to those in brain.

\section{Tau protein is expressed throughout the human and mouse myenteric plexus}

Immunohistochemistry was used to examine the localization of tau proteins in the human and rodent ENS. Human colonic myenteric plexus showed intense tau immunoreactivity in both neuronal cell bodies and processes when pan-tau A0024, 3R and 4R antibodies were used, which nearly completely overlapped with beta-tubulin immunostaining (Fig. 4a).

In order to examine the localization of tau proteins in the ENS of htau and wild-type mice, sections of small intestine (duodenum, jejunum, ileum) and large intestine (proximal colon and distal colon) were dissected from 2-month-old htau, wild-type and tau knockout mice to isolate the myenteric plexus. Tissue was immunolabelled with an antibody against total tau (A0024). eGFP fluorescence was also imaged as it is inserted in tau exon 1 to disrupt tau expression in tau knockout and htau mice [3]. Tau proteins were found to be abundant throughout the GI tract of htau and wild-type mice, including in the duodenum, jejunum, ileum, proximal colon and distal colon (Fig. 4b). There were no apparent differences in neuronal tau localisation between these regions. Htau proximal colon exhibited dense ganglia and axons and a robust tau signal, whereas the axons and ganglia in htau ileum and WT jejunum were less dense and the resulting tau signal was comparatively less intense. Tau KO express GFP which is observed, and show no tau immunoreactivity. Thus, tau protein is expressed in the myenteric plexus throughout the GI tract of wild-type and htau mice.

\section{Tau isoforms are phosphorylated in mature ENS but are not susceptible to dephosphorylation with lambda phosphatase}

The phosphorylation of tau at multiple serine and threonine sites has been described in both developing and adult brain and is the predominant mechanism by which tau functions are regulated [32]. This logically led us to analyze tau phosphorylation in mature human ENS. Two antibodies specific for tau phosphorylated at Ser202/ Thr205 (AT8) [26] and Ser396 (PHF13) [19] detected one single band at $53-54 \mathrm{kDa}$ in colon surgical specimen and biopsies (Fig. 1b and c), thereby demonstrating that the enteric $1 \mathrm{~N} 3 \mathrm{R}$ and $0 \mathrm{~N} 4 \mathrm{R}$ tau isoforms are phosphorylated on serine residues under physiological conditions.

We were nevertheless struck by the fact that, in contrast to the brain, lambda phosphatase treatment did not appear to influence the charge/mobility of tau bands in human colon samples when the pan-Tau antibody A0024 was used (Fig. 1a). To further investigate if tau can be dephosphorylated in adult human ENS, colonic biopsy 
a

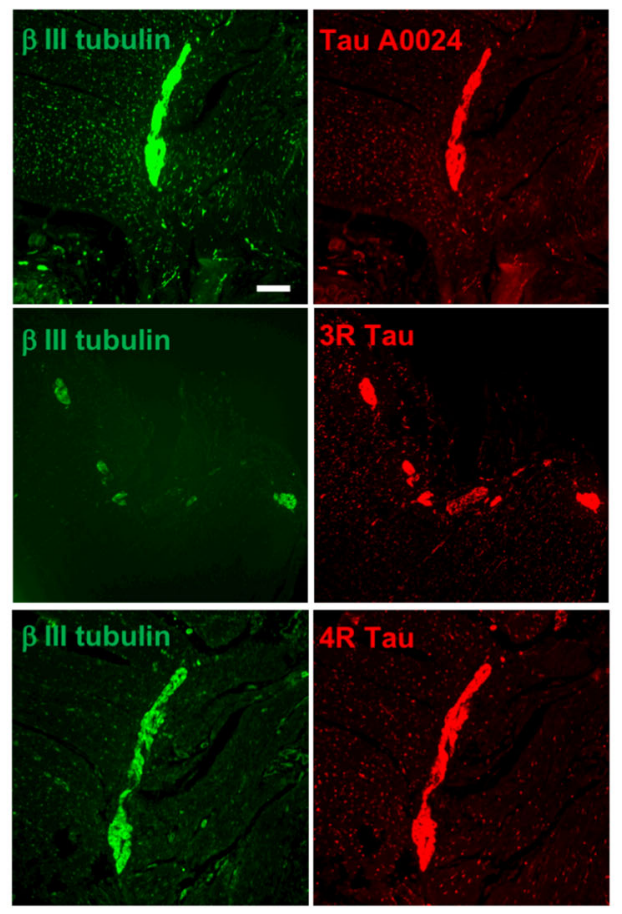

b

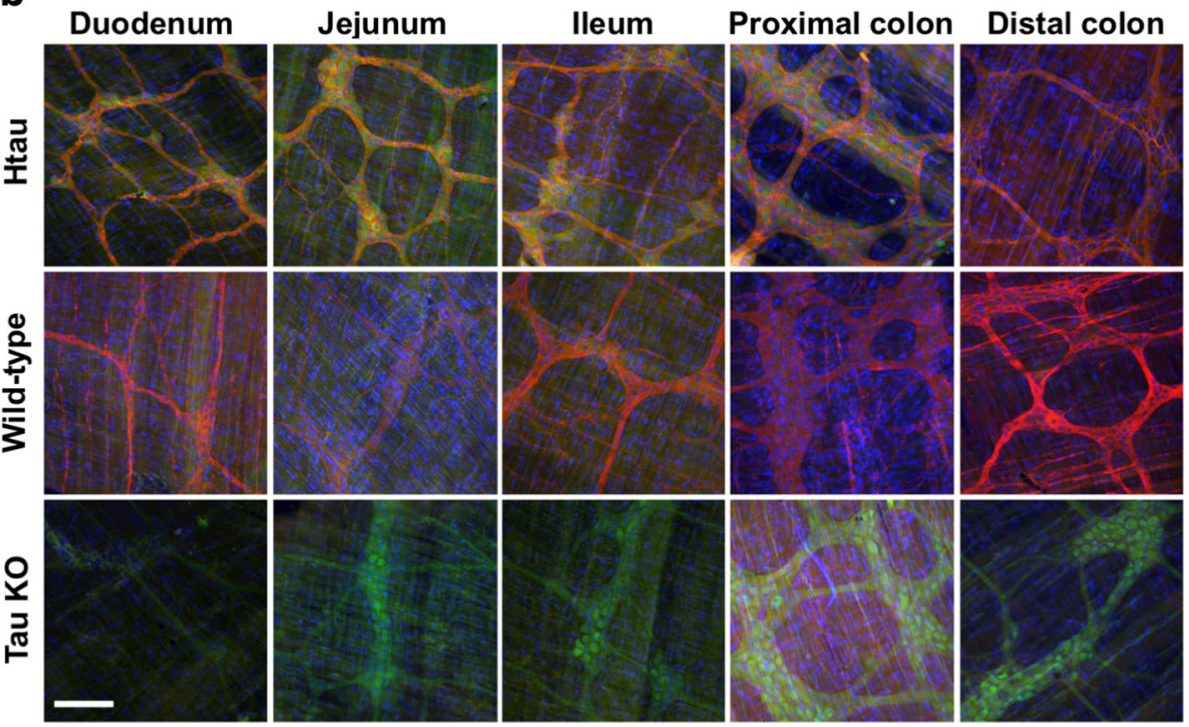

Fig. 4 Distribution and localization of tau in human, htau and wild-type mouse myenteric plexus. a Total tau antibody A0024 and the isoform-specific antibodies against 3R and 4R-tau were used to detect tau in the myenteric plexus of a human colonic sample. An antibody specific to betalll-tubulin was used to specifically label neurons. Scale bar is $200 \mu \mathrm{m}$ (b) Total tau antibody A0024 was used to detect tau in the myenteric plexus of the duodenum, jejunum, ileum, proximal colon and distal colon of 2-month-old htau, wild-type (WT) and tau knockout (KO) mice. Merged images show tau (red), EGFP (green) and Hoechst 33342 labelling of nucleic acids (blue). Scale bar is $100 \mu$ m. Images are representative of three independent experiments

lysates were treated with lambda phosphatase and western blots of these samples were probed with the phospho tau-specific antibodies AT8 (phospho-Ser202/Thr205), PHF1 (phospho-Ser396/404) and Tau-1 (dephospho-Ser199/202/Thr205). Antibodies against ERK were used to check the efficiency of treatment. Although lambda phosphatase efficiently dephosphoylated ERK, it did not modify the phosphorylation state of tau, suggesting that tau is relatively resistant to dephosphorylation in the human adult ENS (Fig. 5a). To further examine tau phosphorylation in mature ENS, we analyzed enteric tau phosphorylation and dephosphorylation in htau mice. Tau is phosphorylated in htau mouse ENS, as phospho-Ser202-positive tau was detected in their 
a

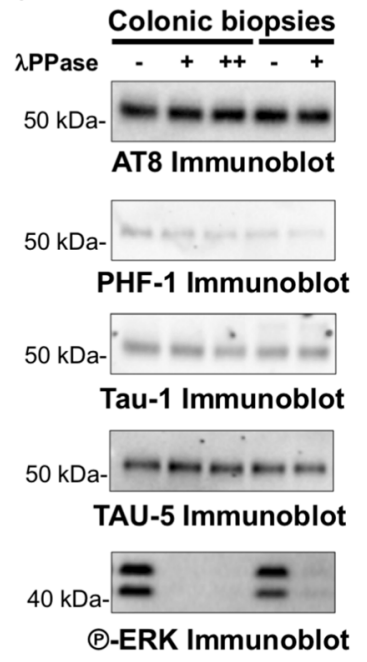

b
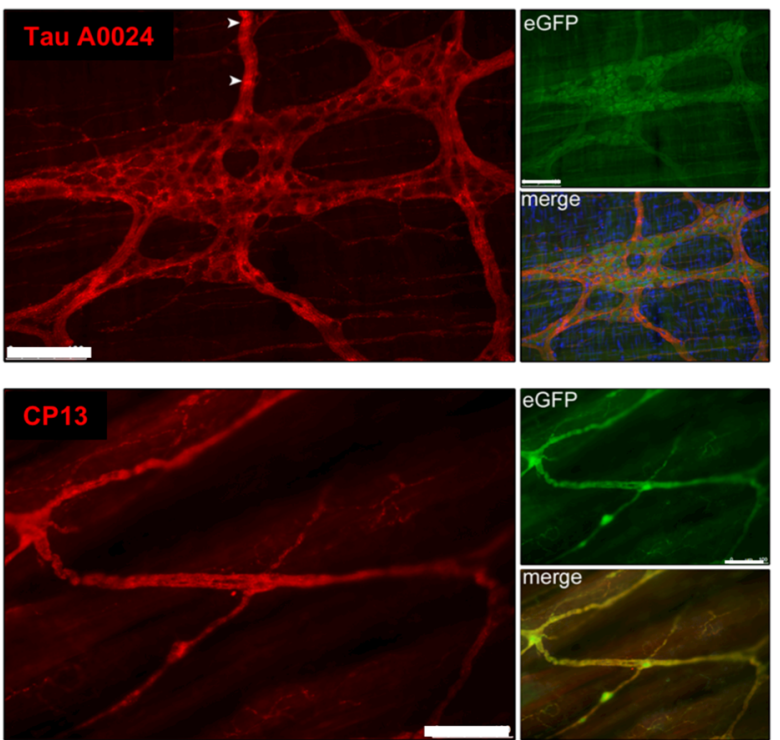

C

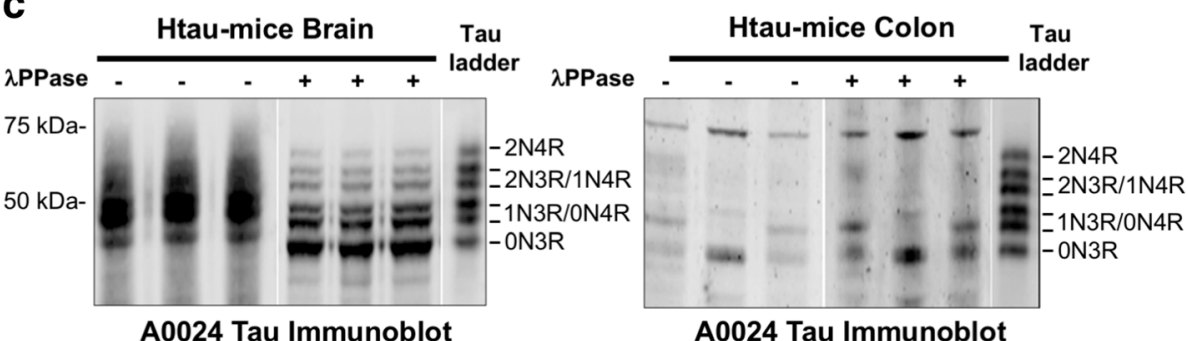

Fig. 5 Poor susceptibility of tau to dephosphorylation in mature ENS. a Sigmoid colon biopsies lysates from 2 control subjects (\#183, 3 first lanes and \#208, 2 last lanes) were subjected to immunoblot analysis using TAU-5, AT8, PHF-1 and Tau-1 and antibodies. Lysates were treated with (+ for $1 \mathrm{~h}$ and ++ for $3 \mathrm{~h}$ ) or without (-) lambda phosphatase before immunoblotting. The effectiveness of dephosphorylation was confirmed by phospho-ERK immunoblot (P-ERK immunoblot). b Antibodies against total tau (Tau antibody A0024) and tau phosphorylated at serine 202 (CP13) (both shown in red) were used to detect tau in the myenteric plexus of the proximal colon from htau mice. eGFP expression is shown in green, together with merged images including Hoechst 33,342 nuclear labelling (blue). Scale bars are $100 \mu \mathrm{m}$. $N=3$. c. Brain and proximal colon homogenates from htau mice were treated with or without lambda phosphatase (+ or -) and immunoblotted with pan-Tau antibody A0024. A recombinant human tau ladder was included on each blot. White lines indicate rearrangement of lanes within the same blot for clarity. Data and images are representative of three independent experiments

colonic myenteric plexus (Fig. 5b). Samples of brain and proximal colon from 2-month-old htau mice were immunoblotted with the pan-Tau antibody A0024. Htau brain showed prominent tau bands ranging from 45 to $70 \mathrm{kDa}$, in agreement with previous reports $[3,52]$. Treatment of these samples with lambda phosphatase to dephosphorylate tau showed that all six major isoforms of tau are expressed in htau brain; these showed good alignment with a recombinant human tau ladder (Fig. 5c). Multiple tau immunoreactive bands ranging from approximately $25-70 \mathrm{kDa}$ were detected in samples from htau proximal colon (Fig. 5c). Two doublets of bands at approximately 45-50 kDa and 55-60 kDa were apparent, which likely corresponds to full-length tau with 0 or $1 \mathrm{~N}$ terminal inserts, likely the $1 \mathrm{~N} 3 \mathrm{R}$ and ON4R isoforms. Moreover, and in contrast to results with htau brain samples, lambda phosphatase treatment did not appear to influence the charge/mobility of tau bands in proximal colon samples (Fig. 5c).

Tau expression levels are unaltered in the ENS in PSP

An increase of the $4 \mathrm{R}$ tau to $3 \mathrm{R}$ tau isoform ratio has been described in some brain regions in PSP [38]. We thus analyzed the expression levels of tau and the relative abundance of $3 R$ and $4 R$ isoforms in the ENS in colonic biopsies from 5 PSP patients in comparison to colonic samples from 10 PD patients and 9 controls devoid of neurodegenerative disorders. Clinical features of the study population are shown in Table 1 . The expression levels of total tau as assessed by immunoblots using the Tau-5 antibody, and the 3R/4R ratio was found 
not to differ between PSP samples and those from PD and controls (Fig. 6a).

\section{Tau phosphorylation and truncation in the ENS are} similar in PSP, PD and control subjects

Abnormal phosphorylation of tau is a characteristic feature of PSP brain $[24,49]$ and we therefore analyzed the phosphorylation state of tau in colonic biopsies from PSP patients using the AT8 and PHF-1 antibodies. There were no apparent alterations in tau phosphorylation at these sites in PSP samples in comparison to those from PD and controls, or between PD and controls (Fig. 6b).

Besides abnormal phosphorylation, tau is also truncated in the pathological deposits observed in tauopathies, and especially in PSP [31, 51]. C-terminal tau truncation by caspase-3 was evaluated using a Tau Asp421 antibody, which is specific for tau cleaved at Asp421, along with an antibody against the extreme C-terminus of tau (TP70) [59]. Quantification of the immunoreactive bands detected by Tau Asp421 and TP70 showed no difference in tau truncation at Asp421 and the presence of an intact C-terminus, between PD, PSP and control subjects (Fig. 6c).

\section{Four tau isoforms are expressed and phosphorylated in primary culture of rat ENS}

Primary neuronal cultures of rat CNS neurons, which primarily express the shortest tau isoforms 0 N3R and $0 N 4 R$, have been widely used for studying tau expression, aggregation and secretion $[13,52,56]$. The brain is not the only source from which neurons can be cultured and there are now established protocols for the isolation of enteric neurons from rodents and especially rats. These have already been shown to be useful for studying the expression of neuronal proteins involved in neurodegeneration such as alpha-synuclein [54], however the expression pattern of tau isoforms in rat primary ENS culture remains to be determined. As a first approach to identify tau isoforms in cultured rat enteric neurons, we compared the banding pattern on western blots of total tau as evaluated with the A0024 pan-Tau antibody between primary culture of ENS and cortical neurons. In keeping with previous observations [13, 52], this antibody detected a tau doublet with one major band at $50 \mathrm{kDa}$ and a fainter one around $53 \mathrm{kDa}$ in CNS neurons, which likely correspond to $0 \mathrm{~N} 3 \mathrm{R}$ and $0 \mathrm{~N} 4 \mathrm{R}$ isoforms, respectively (Fig. 7a). In ENS neurons, the observed banding pattern was markedly different with a triplet of 50,53 and $58 \mathrm{kDa}$ bands observed, the latter showing the most intense labelling (Fig. 7a). Further blotting with the $3 R$ and $4 R$ specific antibodies identified $0 \mathrm{~N} 3 \mathrm{R}, 1 \mathrm{~N} 3 \mathrm{R} / 0 \mathrm{~N} 4 \mathrm{R}$ and $2 \mathrm{~N} 3 \mathrm{R}$ as the main component of the tau triplet observed in primary culture of ENS, while $0 \mathrm{~N} 3 \mathrm{R}$ and $0 \mathrm{~N} 4 \mathrm{R}$ were the two primary tau isoforms expressed by primary culture of CNS (Fig. 7a).
Phosphorylation of tau at multiple serine and threonine sites can be modulated in primary culture of CNS [13]. To determine whether tau phosphorylation can be also regulated in primary culture of rat ENS, we treated the cells with either lambda phosphatase or a combination of serine/threonine phosphatase inhibitors. Treatment with lambda phosphatase caused tau dephosphorylation, as evidenced by a significant downward shift in mobility of the tau triplet detected with either the pan-Tau A0024 or 3R antibodies (Fig. 7b). Conversely, treatment with phosphatase inhibitors induced tau phosphorylation as shown by upward shift in mobility of the protein on Western blots probed with the pan-Tau A0024 antibody, and the disappearance of all immunoreactive bands when the Tau- 1 antibody against dephosphorylated tau was used (Fig. 7c). When the AT8 antibody was used, no signal was observed under basal conditions, while 3 immunoreactive bands were detected in the presence of phosphatase inhibitors (Fig. 7c). The PHF-1 antibody also detected three immunoreactive bands in untreated cells. An increase in signal intensity along with a mobility shift of all 3 bands was observed following treatment of primary ENS cultures with phosphatases inhibitors (Fig. 7c). Thus, the phosphorylation of ENS tau can be modified, at least in an in vitro setting.

\section{$3 R$ and $4 R$ tau are differentially expressed in rat primary enteric neuron cultures}

Lastly, the distribution of tau in rat enteric neurons in culture was examined by immunohistochemistry using pan-Tau A0024, 3R and 4R-tau antibodies at 14 days in vitro. Total tau immunoreactivity was observed in both soma and neuronal processes and the staining patterns produced by pan-Tau A0024, 3R-tau and beta III tubulin antibodies were virtually superimposable (Fig. 8). The $4 \mathrm{R}$-tau staining pattern was markedly different from that observed with 3R-tau and was primarily limited to the cell bodies (Fig. 8). These data indicate that $3 R$ and $4 R$ tau species have different localization in rat primary ENS neurons.

\section{Discussion}

Here, we have used samples of brain and gut from humans, htau transgenic mice and rat primary cultures to show that the isoform profile of tau differs between the ENS and the CNS. We identified 1N3R and ON4R as the two main tau isoforms expressed in adult human ENS and observed an apparent difference in the relative abundance of different tau isoforms in htau gut and brain, with $1 \mathrm{~N}$ and $2 \mathrm{~N}$ tau isoforms being over-represented at mRNA levels in htau gut tissues, although $0 \mathrm{~N}$ and $1 \mathrm{~N}$ isoforms were the predominant protein species detected. We also found that primary culture of rat ENS express four isoforms of tau contrasting with the predominant 

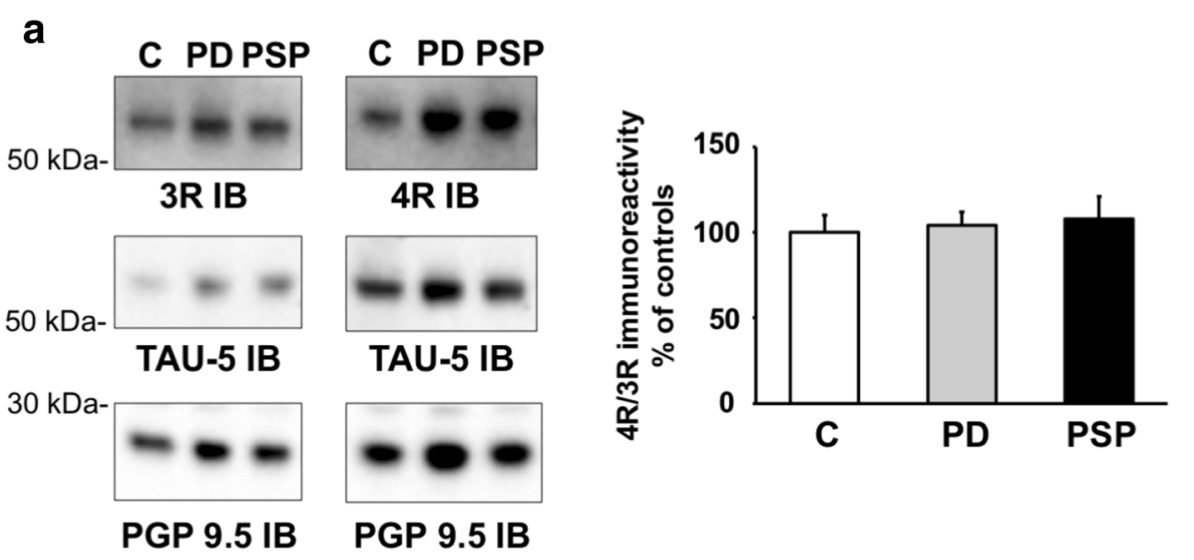

b
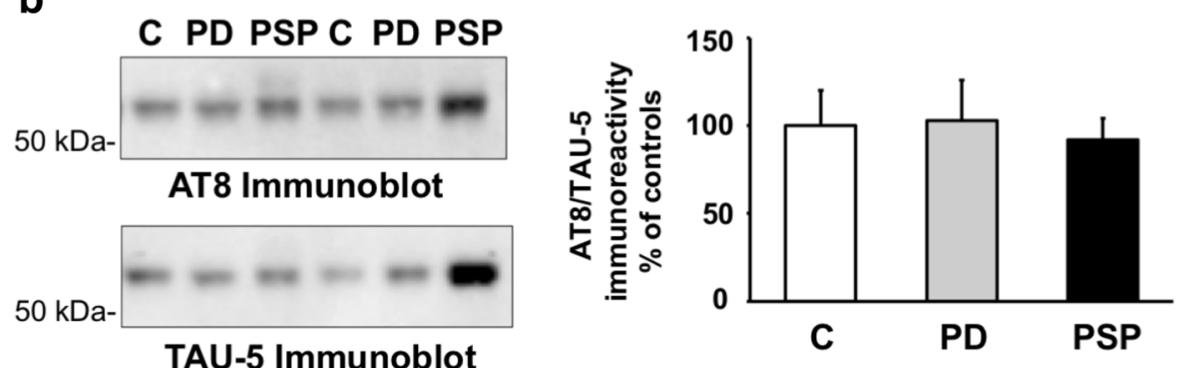

TAU-5 Immunoblot
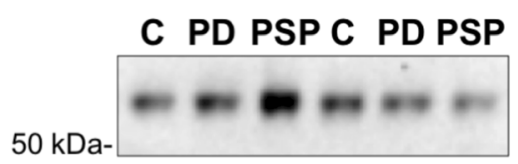

PHF-1 Immunoblot
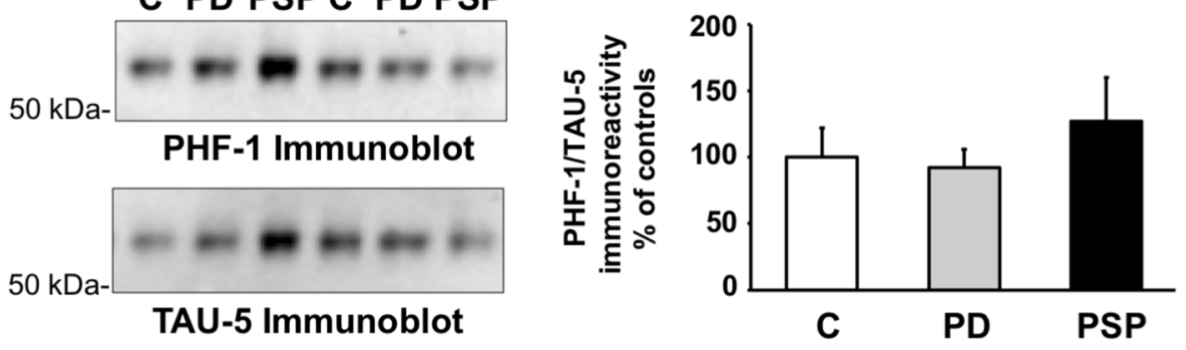

C
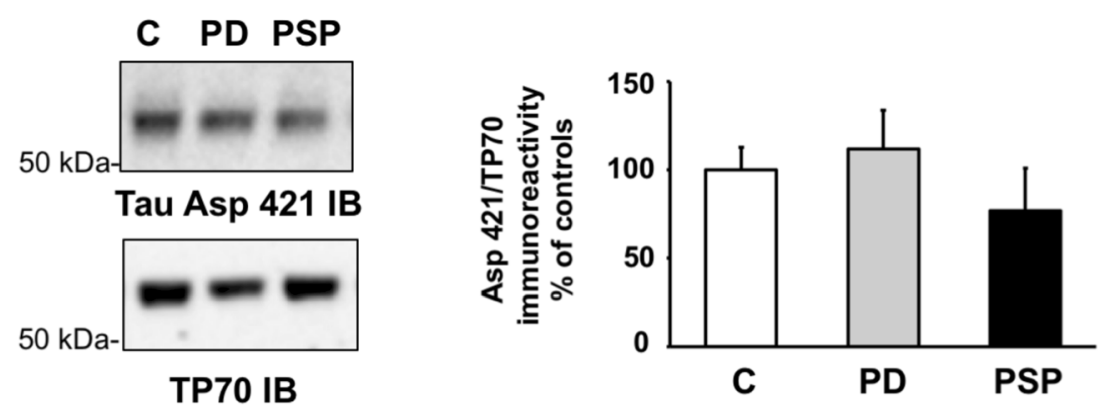

Fig. 6 Tau expression and post-translational modifications in colonic biopsies from patients progressive supranuclear palsy, Parkinson's disease and control subjects. a Biopsies lysates were subjected to immunoblot analysis using antibodies against total tau (TAU-5) and against 3R and 4R isoforms. An antibody against protein gene product (PGP) 9.5 was used as a loading control. For quantification, the optical densities of tau-immunoreactive bands were measured, normalized to the optical densities of PGP9.5 immunoreactive bands in the same samples and expressed as percentages of controls. b Biopsies lysates were subjected to immunoblot analysis using AT8, PHF-1 and TAU-5 antibodies. The optical densities of phospho-tau-immunoreactive bands were measured, normalized to the optical densities of TAU-5 immunoreactive bands in the same samples, expressed as percentages of controls. c Biopsies lysates were subjected to immunoblot analysis using antibodies Tau Asp421 and TP70. The optical densities of immunoreactive bands were measured, normalized to the optical densities of TP70 immunoreactive bands in the same samples, expressed as percentages of controls. Data correspond to mean \pm SEM for 9 control samples (C), 10 from Parkinson's disease (PD) patients and 5 from progressive supranuclear palsy (PSP). Immunoblot (IB) 


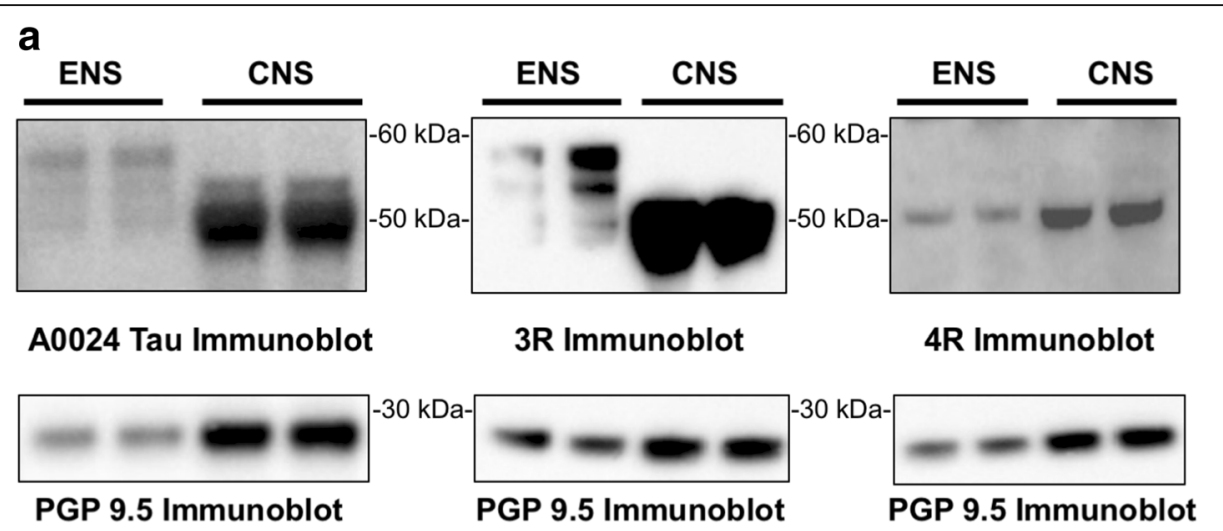

b

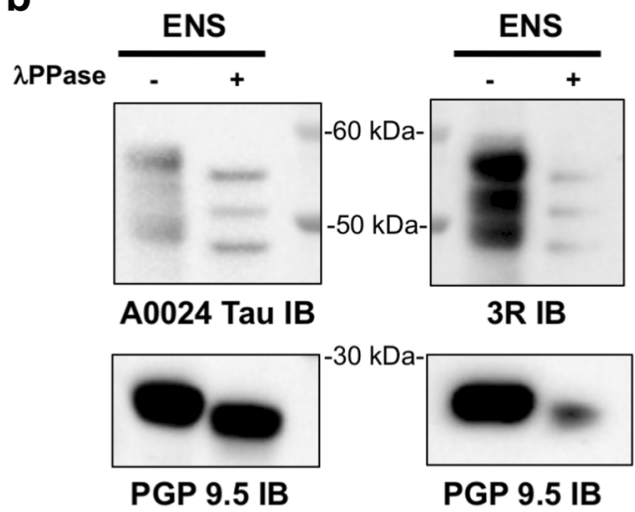

C

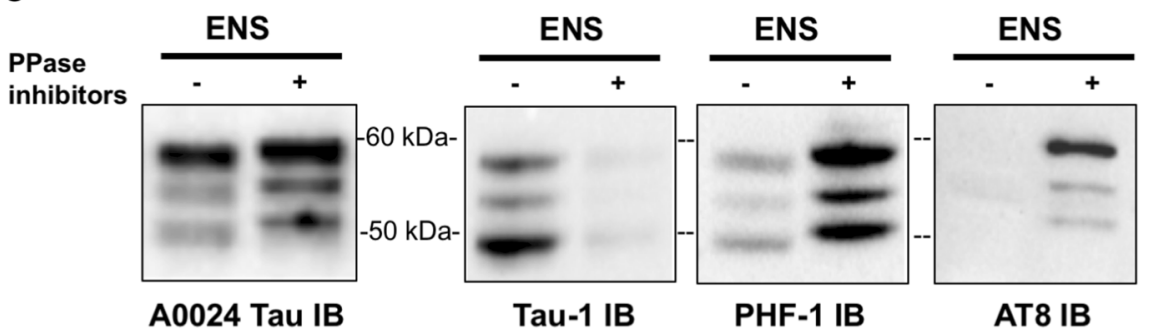

Fig. 7 Tau isoform profile and phosphorylation state in rat primary culture of ENS. a Lysates of rat primary ENS and CNS cultures were subjected to immunoblot analysis using the pan-Tau antibody A0024 and the isoform specific antibodies $3 R$ and 4R. $\mathbf{b}$ Primary culture lysates were treated with (+) or without (-) lambda phosphatase before immunoblot analysis with the pan-Tau antibody A0024 and the isoform specific antibody against 3R-tau. PGP9.5 was used as a loading control. c Primary culture of rat ENS were treated (+) or not (-) with a cocktail of 3 phosphatase inhibitors including $1 \mu \mathrm{M}$ okadaic acid, $1 \mu \mathrm{M}$ ciclosporine $\mathrm{A}$ and $6.75 \mu \mathrm{M}$ sanguinarine (Ppase inhibitors) for $1 \mathrm{~h}$. Fifteen $\mu \mathrm{g}$ of cell lysates were subjected to immunoblot analysis using Tau-1, AT8 and PHF-1 antibodies. IB is for immunoblot. The results shown in (a), (b) and (c) are representative of 2, 4 and 3 independent experiments, respectively

expression of the single fetal tau 0N3R isoform in rat primary cortical neurons. The ENS and the CNS both contain integrated nervous networks and the similarities between them, including between neurons and glia at a morphological level, have led to the ENS being described as the 'brain in the gut' or the 'second brain' [22]. Our current and previous results suggest that this anatomical resemblance does not extend to the molecular level as the ENS expresses only a limited number of isoforms of neuronal and glial markers as compared to the brain [10], although the functional consequences of these differences still remain to be determined.

Tau was found to be expressed in both the myenteric and submucosal plexus of human colon and throughout the ENS of wild-type mice and in the htau mouse model. In both the human and rodent ENS, tau protein had a mainly axonal and somatic distribution, which might be expected since in physiological conditions, tau is described as being a predominantly axonal protein [32]. The presence of nuclear tau has been documented in a wide variety 

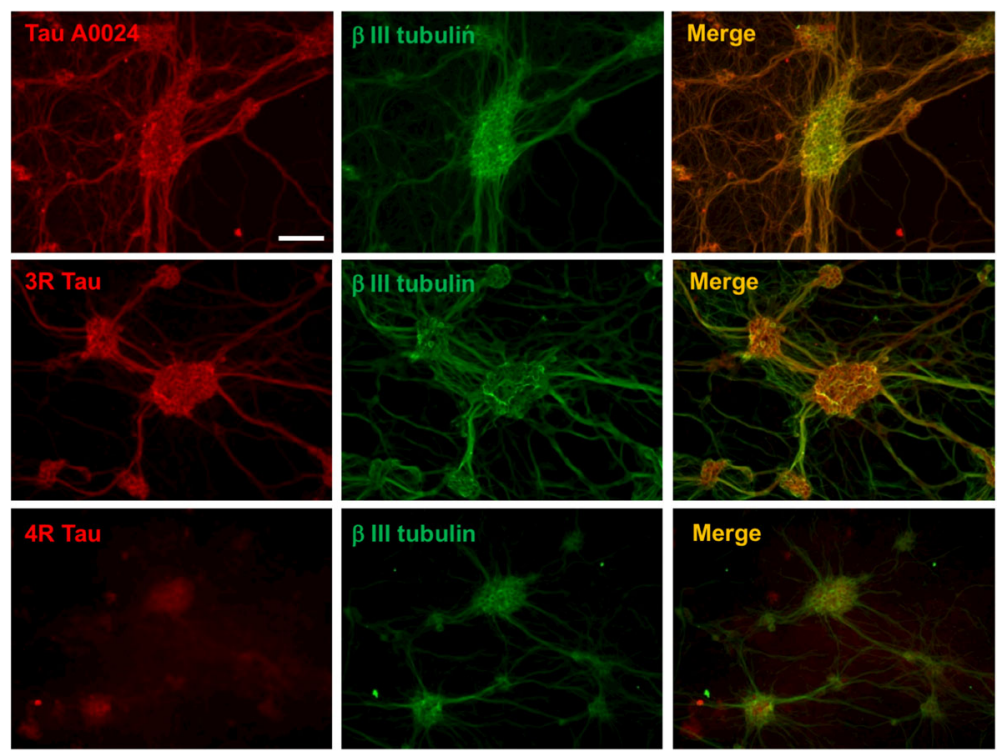

Fig. 8 Distribution and localization of tau in primary culture of rat ENS. After 14 days in culture, primary culture of rat ENS were immunostained with the pan-Tau antibody A0024 and the isoforms specific antibodies against 3R and 4R-tau. Scale bar is $100 \mu \mathrm{M}$

of cell and animal systems, including human and rodent brains and neuronal cell lines (reviewed in [32]). So far, the transcript encoding nuclear tau has not been formally identified but findings obtained in mouse brain suggest that the $1 \mathrm{~N} 4 \mathrm{R}$ isoform is preferentially localised in the nucleus [46]. Although our immunofluorescence experiments performed in mature human and mouse ENS, as well as in primary culture of rat ENS, clearly showed that tau was mainly axonal and somatic, we cannot rule out that a small proportion of enteric tau could also be nuclear. Further experiments including high resolution imaging and biochemical subcellular fractionation will be needed to answer this question.

A panel of well-characterised phospho-specific tau antibodies were used to show that tau is phosphorylated in the ENS of healthy subjects at Ser202/Thr205 and Ser396/ Ser404. Tau is known to be phosphorylated at these sites under physiological conditions, with elevated phosphorylation at these epitopes chacteristic of pathological conditions in the CNS (reviewed in [53]). There is mounting evidence to suggest that tau phosphorylation plays a key role in neuronal physiology. The function of tau is strongly affected by its phosphorylation status, influencing its ability to interact with signaling proteins and kinases [57], its association with microtubules and membranes and its ability to regulate axonal transport [58]. Phosphorylation of Ser202/Thr205 and Ser396/Ser404 is commonly found in primary cortical neurons under basal conditions [2] as well as in snap-frozen brain biopsies from subject devoid of neurodegenerative conditions [47], suggesting that these sites are involved in the normal physiology of the CNS. This is further reinforced by the recent observation showing the presence of endogenous tau phosphorylated at these sites at postsynaptic sites in hippocampal neurons where tau interacts with the PSD95-NMDA receptor complex to regulate synaptic activity [48]. These results obtained in the CNS could be extended to the ENS where neuronal plasticity has also been described following modulation of neuronal activity [9, 37].

Soluble tau from adult human brain consist of a heterogeneous mixture of tau isoforms in multiple states of phosphorylation $[25,28]$. Because normal electrophoresis techniques do not separate the individual tau isoforms, correct identification of the isoform composition of soluble tau requires an efficient dephosphorylation reaction with lambda phosphatase before immunoblotting [35]. Dephosphorylation of tau from normal adult human brain classically produces a downwards shift enabling a more precise separation and identification of the six tau isoforms $[25,35]$. We therefore used the same approach in mature human ENS and the gut of htau mice. In sharp contrast to results with human and htau brain samples, lambda phosphatase treatment did not change the charge/mobility of tau bands in colon samples, suggesting that gut tau may have not been efficiently dephosphorylated. Since western blot and immunohistochemical findings showed that tau in gut is in fact phosphorylated, at least at Ser202, Thr205 and Ser396/ Ser404 this raises the possibility that ENS tau is modified in such a way that it is not susceptible to dephosphorylation. This relative resistance to dephosphorylation, which might be due to conformational changes occurring in case of phosphorylation at some specific sites [18], is specific to adult ENS tau as lambda 
phosphatase efficiently dephosphorylated tau in rat primary ENS cultures prepared from fetal rats.

In 1978 the first study on cultured myenteric neurons was published [40] and since then there has been a growing interest in this method with several different culture preparations being developed. Using primary cultures of rat ENS $[9,11]$, we have shown that fetal rat enteric neurons express four isoforms of tau, including the three $3 \mathrm{R}$ isoforms. This again stands in sharp contrast to the CNS as rat primary cortical neurons primarily express the 0N3R isoform (our study and [13]). We also show that tau isoforms present in primary ENS culture are phosphorylated under basal conditions and their levels of phosphorylation can be down or upregulated. This suggests that cultured ENS might be helpful to study the regulation of tau expression, phosphorylation and secretion not only in physiological conditions but also in the context of enteric neuropathies [55].

We did not observe any pathological tau changes in the ENS of PSP patients. This stands in sharp contrast with the fact that PSP is considered a prototypical tauopathy of the CNS characterized by tau hyperphosphorylation and truncation [31, 67] and an imbalance in 4R/3R ratio [38]. We have recently proposed that the ENS may be a mirror on to the PD pathology of the CNS since it recapitulates several of the neuronal and glial changes observed in the brain $[10,15,45]$. Our results suggest that, unlike PD, the pathological process in PSP is limited to the CNS and does not involve the ENS. This is supported by the paucity of studies reporting that the peripheral nervous systems are affected in PSP (reviewed in [64]) and by our observation of a lack of glial reaction in the gut in PSP patients [10]. One obvious limitation of this work is that our analysis of PSP samples was restricted to the analysis of the submucosal plexus. We can therefore not rule out that the absence of overt pathological changes in tau in colonic samples from our PSP patients may be due to this limited regional analysis and perhaps different findings would have been obtained had we examined the myenteric plexus. The refinement of new endoscopic procedures, such as full thickness biopsies [50], which provide access to both myenteric and submucosal plexi, may help to answer these critical questions. A second limitation in our study is the lack of neuropathological confirmation of PD and PSP, as the clinical diagnosis of both disorders may have a relatively poor accuracy $[1,42]$, especially for PD patients for whom signs and symptoms have been present for less than 5 years [1]. In addition, we can not rule out that some of our control subjects may have asymptomatic tauopathy [12].

\section{Conclusions}

We have characterised tau in the human and rodent ENS under physiological conditions and tauopathies. We show differences in tau isoform expression at mRNA and protein level, and in the susceptibility of tau to be dephosphorylated in the CNS and ENS. The data we have acquired on tau in the ENS strongly supports additional future studies aimed at expanding our knowledge of peripheral pathology in neurodegenrative disorders of the CNS and in enteric neuropathies [14].

\section{Additional file}

Additional file 1: Figure S1. Validation of the Cosmo-bio 4R antibody. (PDF 220 kb)

\section{Abbreviations}

AD: Alzheimer's disease; BSA: Bovine serum albumen; CNS: Central nervous system; ENS: Enteric nervous system; ERK: Extracellular signal-regulated kinases; Gl: Gastrointestinal; KO: Knockout; MP: Myenteric plexus; PCR: Polymerase chain reaction; PD: Parkinson's disease; PSP: Progressive supranuclear palsy; Ser: Serine; SMP: Submucosal plexus; TBS: Tris-buffered saline; Thr: Threonine

\section{Acknowledgements}

We are grateful to Professor Peter Davies (Feinstein Institute for Medical Research, NY, USA) for his generous gift of tau antibodies.

\section{Funding}

This work was supported by BBSRC/AstraZeneca (BB/L502601/1 to WN), the National Centre for the Replacement, Refinement and Reduction of Animals in Research (NC3Rs, NC/K500343/1 to WN), CECAP, FFGP and PSP France (to AP and PD).

\section{Availability of data and materials}

The datasets used and/or analysed during the current study available from the corresponding author on reasonable request.

\section{Authors' contributions}

AL, MAW, AGC, AP, SP, MT and JG performed the experiments and analyzed the data. ED and MRD managed the biobanking and dissected the colonic samples. ED and EC performed the endoscopy. MAW, PD, MN, DPH, MSP and WN designed the research and MAW, WN and PD wrote the manuscript. All authors read and approved the final manuscript.

\section{Ethics approval and consent to participate}

All housing and experimental procedures were carried out in compliance with the local ethical review panel of King's College London under a UK Home Office project license held in accordance with the Animals (Scientific Procedures) Act 1986 and the European Directive 2010/63/EU. Regarding sigmoid biopsies sampling, the study protocol was approved by the local Committee on Ethics and Human Research (Comité de Protection des Personnes Ouest VI), and registered on ClinicalTrials.gov (EnteroLark and ColoBioParker, identifier NCT01618383 and NCT01353183, respectively). Written informed consent was obtained from each patient and from each normal volunteer.

\section{Consent for publication \\ Not applicable.}

\section{Competing interests}

Michael S. Perkinton is an employee of Medlmmune.

\section{Publisher's Note}

Springer Nature remains neutral with regard to jurisdictional claims in published maps and institutional affiliations.

\section{Author details}

${ }^{1}$ Inserm, U1235, 1 rue Gaston Veil, F-44035 Nantes, France. ${ }^{2}$ Department of Neurology, CHU Nantes, F-44093 Nantes, France. ${ }^{3}$ University Nantes, F-44000 
Nantes, France. ${ }^{4}$ King's College London, Institute of Psychiatry, Psychology and Neuroscience, Department of Basic and Clinical Neuroscience, Maurice Wohl Clinical Neuroscience Institute, Rm 1.23, 5 Cutcombe Road, Camberwell, London SE5 9RX, UK. ${ }^{5}$ Neuroscience, IMED Biotech Unit, AstraZeneca, Cambridge CB21 6GH, UK.

Received: 14 June 2018 Accepted: 6 July 2018

Published online: 23 July 2018

\section{References}

1. Adler CH, Beach TG, Hentz JG, Shill HA, Caviness JN, Driver-Dunckley E et al (2014) Low clinical diagnostic accuracy of early vs advanced Parkinson disease: clinicopathologic study. Neurology 83:406-412. https://doi.org/10. 1212/WNL.000000000000064

2. Anderton BH, Brion JP, Couck AM, Davis DR, Gallo JM, Hanger DP et al (1995) Modulation of PHF-like tau phosphorylation in cultured neurones and transfected cells. Neurobiol Aging 16:389-397 discussion 398-402

3. Andorfer C, Kress Y, Espinoza M, de Silva R, Tucker KL, Barde Y-A et al (2003) Hyperphosphorylation and aggregation of tau in mice expressing normal human tau isoforms. J Neurochem 86:582-590

4. Andreadis A (2005) Tau gene alternative splicing: expression patterns, regulation and modulation of function in normal brain and neurodegenerative diseases. Biochim Biophys Acta 1739:91-103. https://doi. org/10.1016/j.bbadis.2004.08.010

5. Barrenschee M, Zorenkov D, Böttner M, Lange C, Cossais F, Scharf AB et al (2017) Distinct pattern of enteric phospho-alpha-synuclein aggregates and gene expression profiles in patients with Parkinson's disease. Acta Neuropathol Commun 5:1. https://doi.org/10.1186/s40478-016-0408-2

6. Beach TG, Adler CH, Sue LI, Vedders L, Lue L, White lii CL et al (2010) Multiorgan distribution of phosphorylated alpha-synuclein histopathology in subjects with Lewy body disorders. Acta Neuropathol 119:689-702. https:// doi.org/10.1007/s00401-010-0664-3

7. Bussière T, Hof PR, Mailliot C, Brown CD, Caillet-Boudin ML, Perl DP et al (1999) Phosphorylated serine422 on tau proteins is a pathological epitope found in several diseases with neurofibrillary degeneration. Acta Neuropathol 97:221-230

8. Chambonnière ML, Mosnier-Damet M, Mosnier JF (2001) Expression of microtubule-associated protein tau by gastrointestinal stromal tumors. Hum Pathol 32:1166-1173

9. Chevalier J, Derkinderen P, Gomes P, Thinard R, Naveilhan P, Vanden Berghe P. Neunlist M (2008) Activity-dependent regulation of tyrosine hydroxylase expression in the enteric nervous system. J Physiol Lond 586:1963-1975. https://doi.org/10.1113/jphysiol.2007.149815

10. Clairembault T, Kamphuis W, Leclair-Visonneau L, Rolli-Derkinderen M, Coron E, Neunlist M et al (2014) Enteric GFAP expression and phosphorylation in Parkinson's disease. J Neurochem 130:805-815. https://doi.org/10.1111/jnc.12742

11. Coquenlorge S, Duchalais E, Chevalier J, Cossais F, Rolli-Derkinderen M, Neunlist M (2014) Modulation of lipopolysaccharide-induced neuronal response by activation of the enteric nervous system. J Neuroinflammation 11:202. https://doi.org/10.1186/s12974-014-0202-7

12. Crary JF, Trojanowski JQ, Schneider JA, Abisambra JF, Abner EL, Alafuzoff I et al (2014) Primary age-related tauopathy (PART): a common pathology associated with human aging. Acta Neuropathol 128:755-766. https://doi. org/10.1007/s00401-014-1349-0

13. Davis DR, Brion JP, Couck AM, Gallo JM, Hanger DP, Ladhani K et al (1995) The phosphorylation state of the microtubule-associated protein tau as affected by glutamate, colchicine and beta-amyloid in primary rat cortical neuronal cultures. Biochem J 309(Pt 3):941-949

14. De Giorgio R, Bianco F, Latorre R, Caio G, Clavenzani P, Bonora E (2016) Enteric neuropathies: yesterday, today and tomorrow. Adv Exp Med Bio 891:123-133. https://doi.org/10.1007/978-3-319-27592-5_12

15. Devos D, Lebouvier T, Lardeux B, Biraud M, Rouaud T, Pouclet H et al (2013) Colonic inflammation in Parkinson's disease. Neurobiol Dis 50:42-48. https:// doi.org/10.1016/j.nbd.2012.09.007

16. Duff K, Knight H, Refolo LM, Sanders S, Yu X, Picciano M et al (2000) Characterization of pathology in transgenic mice over-expressing human genomic and cDNA tau transgenes. Neurobiol Dis 7:87-98. https://doi.org/ 10.1006/nbdi.1999.0279

17. Dugger BN, Whiteside CM, Maarouf CL, Walker DG, Beach TG, Sue LI et al (2016) The presence of select tau species in human peripheral tissues and their relation to Alzheimer's disease. J Alzheimers Dis 51:345-356. https:// doi.org/10.3233/JAD-150859

18. Dupont-Wallois L, Sautière PE, Cocquerelle C, Bailleul B, Delacourte A, Caillet-Boudin ML (1995) Shift from fetal-type to Alzheimer-type phosphorylated tau proteins in SKNSH-SY 5 Y cells treated with okadaic acid. FEBS Lett 357:197-201

19. Ercan E, Eid S, Weber C, Kowalski A, Bichmann M, Behrendt A et al (2017) A validated antibody panel for the characterization of tau posttranslational modifications. Mol Neurodegener 12:87. https://doi.org/10. 1186/s13024-017-0229-1

20. Fischer D, Mukrasch MD, Biernat J, Bibow S, Blackledge M, Griesinger C et al (2009) Conformational changes specific for pseudophosphorylation at serine 262 selectively impair binding of tau to microtubules. Biochemistry 48: 10047-10055. https://doi.org/10.1021/bi901090m

21. Gelpi E, Navarro-Otano J, Tolosa E, Gaig C, Compta Y, Rey MJ et al (2014) Multiple organ involvement by alpha-synuclein pathology in Lewy body disorders. Mov Disord 29:1010-1018. https://doi.org/10.1002/mds.25776

22. Gershon MD (1999) The enteric nervous system: a second brain. Hosp Pract (1995) 34:31-32 35-38, 41-42 passim

23. Giancola F, Torresan F, Repossi R, Bianco F, Latorre R, loannou A et al (2017) Downregulation of neuronal vasoactive intestinal polypeptide in Parkinson's disease and chronic constipation. Neurogastroenterol Motil 29. https://doi. org/10.1111/nmo.12995

24. Gibb GM, de Silva R, Revesz T, Lees AJ, Anderton BH, Hanger DP (2004) Differential involvement and heterogeneous phosphorylation of tau isoforms in progressive supranuclear palsy. Brain Res Mol Brain Res 121:95-101. https:// doi.org/10.1016/j.molbrainres.2003.11.007

25. Goedert M, Jakes R (1990) Expression of separate isoforms of human tau protein: correlation with the tau pattern in brain and effects on tubulin polymerization. EMBO J 9:4225-4230

26. Goedert M, Jakes R, Vanmechelen E (1995) Monoclonal antibody AT8 recognises tau protein phosphorylated at both serine 202 and threonine 205. Neurosci Lett 189:167-169

27. Goedert M, Spillantini MG, Crowther RA (1992) Cloning of a big tau microtubule-associated protein characteristic of the peripheral nervous system. Proc Natl Acad Sci U S A 89:1983-1987

28. Goedert M, Spillantini MG, Jakes R, Rutherford D, Crowther RA (1989) Multiple isoforms of human microtubule-associated protein tau: sequences and localization in neurofibrillary tangles of Alzheimer's disease. Neuron 3:519-526

29. Grundke-labal I, labal K, Tung YC, Quinlan M, Wisniewski HM, Binder LI (1986) Abnormal phosphorylation of the microtubule-associated protein tau (tau) in Alzheimer cytoskeletal pathology. Proc Natl Acad Sci U S A 83:4913-4917

30. Gu Y, Oyama F, Ihara Y (1996) Tau is widely expressed in rat tissues. J Neurochem 67:1235-1244

31. Guillozet-Bongaarts AL, Glajch KE, Libson EG, Cahill ME, Bigio E, Berry RW, Binder LI (2007) Phosphorylation and cleavage of tau in non-AD tauopathies. Acta Neuropathol 113:513-520. https://doi.org/10.1007/s00401007-0209-6

32. Guo T, Noble W, Hanger DP (2017) Roles of tau protein in health and disease. Acta Neuropathol 133:665-704. https://doi.org/10.1007/s00401-017-1707-9

33. Haïk S, Faucheux BA, Sazdovitch V, Privat N, Kemeny J-L, Perret-Liaudet A, Hauw J-J (2003) The sympathetic nervous system is involved in variant Creutzfeldt-Jakob disease. Nat Med 9:1121-1123. https://doi.org/10.1038/ nm922

34. Hanger DP, Byers HL, Wray S, Leung K-Y, Saxton MJ, Seereeram A, Reynolds $\mathrm{CH}$ et al (2007) Novel phosphorylation sites in tau from Alzheimer brain support a role for casein kinase 1 in disease pathogenesis. J Biol Chem 282: 23645-23654. https://doi.org/10.1074/jbc.M703269200

35. Hanger DP, Gibb GM, de Silva R, Boutajangout A, Brion J-P, Revesz T et al (2002) The complex relationship between soluble and insoluble tau in tauopathies revealed by efficient dephosphorylation and specific antibodies. FEBS Lett 531:538-542

36. Heumüller-Klug S, Sticht C, Kaiser K, Wink E, Hagl C, Wessel L, Schäfer K-H (2015) Degradation of intestinal mRNA: a matter of treatment. World J Gastroenterol 21:3499-3508. https://doi.org/10.3748/wjg.v21.i12.3499

37. Hons IM, Storr MA, Mackie K, Lutz B, Pittman QJ, Mawe GM, Sharkey KA (2012) Plasticity of mouse enteric synapses mediated through endocannabinoid and purinergic signaling. Neurogastroenterol Motil 24 e113-e124. https://doi.org/10.1111/j.1365-2982.2011.01860.x

38. Ingelsson M, Ramasamy K, Russ C, Freeman SH, Orne J, Raju S et al (2007) Increase in the relative expression of tau with four microtubule binding 
repeat regions in frontotemporal lobar degeneration and progressive supranuclear palsy brains. Acta Neuropathol 114:471-479. https://doi.org/10. 1007/s00401-007-0280-z

39. Ishizawa T, Mattila P, Davies P, Wang D, Dickson DW (2003) Colocalization of tau and alpha-synuclein epitopes in Lewy bodies. J Neuropathol Exp Neurol 62:389-397

40. Jessen KR, McConnell JD, Purves RD, Burnstock G, Chamley-Campbell J (1978) Tissue culture of mammalian enteric neurons. Brain Res 152:573-579

41. Joiner S, Linehan JM, Brandner S, Wadsworth JDF, Collinge J (2005) High levels of disease related prion protein in the ileum in variant CreutzfeldtJakob disease. Gut 54:1506-1508. https://doi.org/10.1136/gut.2005.072447

42. Joutsa J, Gardberg M, Röyttä M, Kaasinen V (2014) Diagnostic accuracy of parkinsonism syndromes by general neurologists. Parkinsonism Relat Disord 20:840-844. https://doi.org/10.1016/j.parkreldis.2014.04.019

43. Kovacs GG (2017) Tauopathies. Handb Clin Neurol 145:355-368. https://doi org/10.1016/B978-0-12-802395-2.00025-0

44. Lebouvier T, Coron E, Chaumette T, Paillusson S, Bruley d, Varannes S, Neunlist M, Derkinderen P (2010) Routine colonic biopsies as a new tool to study the enteric nervous system in living patients. Neurogastroenterol Motil 22:e11-e14. https://doi.org/10.1111/j.1365-2982.2009.01368.x

45. Lebouvier T, Neunlist M, Bruley d, Varannes S, Coron E, Drouard A, N'Guyen J-M et al (2010) Colonic biopsies to assess the neuropathology of Parkinson's disease and its relationship with symptoms. PLoS One 5:e12728. https://doi.org/10.1371/journal.pone.0012728

46. Liu C, Götz J (2013) Profiling murine tau with $0 \mathrm{~N}, 1 \mathrm{~N}$ and $2 \mathrm{~N}$ isoformspecific antibodies in brain and peripheral organs reveals distinct subcellular localization, with the $1 \mathrm{~N}$ isoform being enriched in the nucleus. PLoS One 8:e84849. https://doi.org/10.1371/journal.pone.0084849

47. Matsuo ES, Shin RW, Billingsley ML, Van deVoorde A, O'Connor M, Trojanowski JQ, Lee VM (1994) Biopsy-derived adult human brain tau is phosphorylated at many of the same sites as Alzheimer's disease paired helical filament tau. Neuron 13:989-1002

48. Mondragón-Rodríguez S, Trillaud-Doppia E, Dudilot A, Bourgeois C, Lauzon M, Leclerc N, Boehm J (2012) Interaction of endogenous tau protein with synaptic proteins is regulated by N-methyl-D-aspartate receptor-dependent tau phosphorylation. J Biol Chem 287:32040-32053. https://doi.org/10.1074/ jbc.M112.401240

49. Morris HR, Gibb G, Katzenschlager R, Wood NW, Hanger DP, Strand C et al (2002) Pathological, clinical and genetic heterogeneity in progressive supranuclear palsy. Brain 125:969-975. https://doi.org/10.1093/brain/awf109

50. Neunlist M, Coquenlorge S, Aubert P, Duchalais-Dassonneville E, des Varannes SB, Meurette G, Coron E (2011) Colonic endoscopic full-thickness biopsies: from the neuropathological analysis of the myenteric plexus to the functional study of neuromuscular transmission. Gastrointest Endosc 73: 1029-1034. https://doi.org/10.1016/j.gie.2011.01.041

51. Newman J, Rissman RA, Sarsoza F, Kim RC, Dick M, Bennett DA et al (2005) Caspase-cleaved tau accumulation in neurodegenerative diseases associated with tau and alpha-synuclein pathology. Acta Neuropathol 110:135-144. https://doi.org/10.1007/s00401-005-1027-3

52. Noble W, Garwood C, Stephenson J, Kinsey AM, Hanger DP, Anderton BH (2009) Minocycline reduces the development of abnormal tau species in models of Alzheimer's disease. FASEB J 23:739-750. https://doi.org/10.1096/ fj.08-113795

53. Noble W, Hanger DP, Miller CCJ, Lovestone S (2013) The importance of tau phosphorylation for neurodegenerative diseases. Front Neurol 4:83. https:// doi.org/10.3389/fneur.2013.00083

54. Paillusson S, Tasselli M, Lebouvier T, Mahé MM, Chevalier J, Biraud M et al (2010) a-Synuclein expression is induced by depolarization and cyclic AMP in enteric neurons. J Neurochem 115:694-706. https://doi.org/10.1111/j. 1471-4159.2010.06962x

55. Phillips RJ, Walter GC, Ringer BE, Higgs KM, Powley TL (2009) Alphasynuclein immunopositive aggregates in the myenteric plexus of the aging Fischer 344 rat. Exp Neurol 220:109-119. https://doi.org/10.1016/j.expneurol. 2009.07.025

56. Pooler AM, Usardi A, Evans CJ, Philpott KL, Noble W, Hanger DP (2012) Dynamic association of tau with neuronal membranes is regulated by phosphorylation. Neurobiol Aging 33:431.e27-431.e38. https://doi.org/10. 1016/j.neurobiolaging.2011.01.005

57. Reynolds CH, Garwood CJ, Wray S, Price C, Kellie S, Perera T et al (2008) Phosphorylation regulates tau interactions with Src homology 3 domains of phosphatidylinositol 3-kinase, phospholipase Cgamma1, Grb2, and Src family kinases. J Biol Chem 283:18177-18186. https://doi.org/10.1074/jbc M709715200

58. Rodríguez-Martín T, Cuchillo-lbáñez I, Noble W, Nyenya F, Anderton BH, Hanger DP (2013) Tau phosphorylation affects its axonal transport and degradation. Neurobiol Aging 34:2146-2157. https://doi.org/10.1016/j. neurobiolaging.2013.03.015

59. Takuma H, Arawaka S, Mori H (2003) Isoforms changes of tau protein during development in various species. Brain Res Dev Brain Res 142:121-127

60. Taleghany N, Oblinger MM (1992) Regional distribution and biochemical characteristics of high molecular weight tau in the nervous system. J Neurosci Res 33:257-265. https://doi.org/10.1002/jnr.490330209

61. Tam PK (1990) An immunohistological study of the human enteric nervous system with microtubule-associated proteins. Gastroenterology 99:1841-1844

62. Tasselli M, Chaumette T, Paillusson S, Monnet Y, Lafoux A, Huchet-Cadiou C et al (2013) Effects of oral administration of rotenone on gastrointestinal functions in mice. Neurogastroenterol Motil 25:e183-e193. https://doi.org/ 10.1111/nmo.12070

63. Usardi A, Pooler AM, Seereeram A, Reynolds CH, Derkinderen P, Anderton B et al (2011) Tyrosine phosphorylation of tau regulates its interactions with Fyn SH2 domains, but not SH3 domains, altering the cellular localization of tau. FEBS J 278:2927-2937. https://doi.org/10.1111/j.1742-4658.2011.08218.x

64. Wakabayashi K, Mori F, Tanji K, Orimo S, Takahashi H (2010) Involvement of the peripheral nervous system in synucleinopathies, tauopathies and other neurodegenerative proteinopathies of the brain. Acta Neuropathol 120:1-12. https://doi.org/10.1007/s00401-010-0706-x

65. Wakabayashi K, Takahashi H, Takeda S, Ohama E, Ikuta F (1988) Parkinson's disease: the presence of Lewy bodies in Auerbach's and Meissner's plexuses. Acta Neuropathol 76:217-221

66. Wills J, Credle J, Haggerty T, Lee J-H, Oaks AW, Sidhu A (2011) Tauopathic changes in the striatum of A53T a-synuclein mutant mouse model of Parkinson's disease. PLoS One 6:e17953. https://doi.org/10.1371/journal. pone.0017953

67. Wray S, Saxton M, Anderton BH, Hanger DP (2008) Direct analysis of tau from PSP brain identifies new phosphorylation sites and a major fragment of $\mathrm{N}$ terminally cleaved tau containing four microtubule-binding repeats. J Neurochem 105:2343-2352. https://doi.org/10.1111/j.1471-4159.2008.05321.x

\section{Ready to submit your research? Choose BMC and benefit from:}

- fast, convenient online submission

- thorough peer review by experienced researchers in your field

- rapid publication on acceptance

- support for research data, including large and complex data types

- gold Open Access which fosters wider collaboration and increased citations

- maximum visibility for your research: over $100 \mathrm{M}$ website views per year

At BMC, research is always in progress.

Learn more biomedcentral.com/submissions 